\title{
Inventory Policies for a Make-to-Order System with a Perishable Component and Fixed Ordering Cost
}

\author{
Katia C. Frank, ${ }^{1}$ Hyun-Soo Ahn, ${ }^{2}$ Rachel Q. Zhang ${ }^{3}$ \\ ${ }^{1}$ Macy's Systems and Technology, Johns Creek, Georgia 30097 \\ ${ }^{2}$ Ross School of Business, University of Michigan, Ann Arbor, Michigan 48109 \\ ${ }^{3}$ Department of Industrial Engineering and Logistics Management, Hong Kong University of Science and Technology, \\ Clear Water Bay, Kowloon, Hong Kong
}

Received 11 January 2007; revised 26 August 2008; accepted 27 September 2008

DOI 10.1002/nav.20332

Published online 20 January 2009 in Wiley InterScience (www.interscience.wiley.com).

\begin{abstract}
We consider a make-to-order production system where two major components, one nonperishable (referred to as part 1) and one perishable (part 2), are needed to fulfill a customer order. In each period, replenishment decisions for both parts need to be made jointly before demand is realized and a fixed ordering cost is incurred for the nonperishable part. We show that a simple $\left(s_{n}, S_{n}^{1}, S_{n}^{2}\right)$ policy is optimal. Under this policy, $S_{n}^{2}$ along with the number of backorders at the beginning of a period if any and the availability of the nonperishable part (part 1) determines the optimal order quantity of the perishable part (part 2), while $\left(s_{n}, S_{n}^{1}\right)$ guide when and how much of part 1 to order at each state. Numerical study demonstrates that the benefits of using the joint replenishment policy can be substantial, especially when the unit costs are high and/or the profit margin is low. (C) 2009 Wiley Periodicals, Inc. Naval Research Logistics 56: 127-141, 2009
\end{abstract}

Keywords: inventory; dynamic programming; stochastic

\section{INTRODUCTION}

In food, chemical, and pharmaceutical industries, many products are made using both perishable and nonperishable components (or ingredients). In a mail-order bakery company where orders are produced and shipped in the make-to-order fashion, a final product is made from both perishable ingredients (e.g., eggs, milk, and fruits) and relatively long lasting ingredients and components (e.g., packaging materials, sugar, and baking powder). In chemical or pharmaceutical industries, some intermediate or final chemical compounds are produced by combining both chemically unstable (perishable) and other non-perishable ingredients.

In this article, we consider a make-to-order production system where the final product is comprised of two major components, part 1 and part 2. Although a part 2 item is perishable and lasts only one period, a part 1 item has relatively long shelf life and hence is treated as nonperishable. We assume zero replenishment lead time for both parts. Once demand is realized, production starts and all customers are

Correspondence to: H.-S. Ahn (hsahn@bus.umich.edu) willing to wait. Although part 1 has an infinite lifetime and incurs a fixed ordering cost whenever an order is placed, part 2 perishes after one period and has no fixed ordering cost associated with it. We do not explicitly consider a fixed ordering cost for the perishable part for the following two reasons. First, production cannot start without either part and an order of the perishable part is required for each production run anyway. Second, in the case where this fixed ordering cost is significant and it is not profitable to schedule production in every period, one can adjust the length of the periods. However, part 1 has a long shelf life and a fixed ordering cost needs to be considered.

At first glance, one can make inventory decisions for each part in isolation, and coordinate the replenishment in implementation. That is, one first finds the order-up-to levels for the perishable component (part 2) by solving a series of newsvendor problems and then specifies $(s, S)$ for the nonperishable part (part 1) using known techniques. In each period, one makes sure that the number of part 2 items does not exceed the number of part 1 items. Although each policy is optimal in its corresponding single item system under general conditions, there are two difficulties with this approach. First, because 
production can start and a demand can be fulfilled only when both parts are available, the underage costs for calculating the order-up-to levels for part 2 clearly depend on the availability of part 1 items and are no longer constant. Therefore, the ordering decision on part 2 depends on that of part 1 in each period and the traditional newsvendor model for perishable products does not apply directly. Second, decisions on when to order part 1 and how much to order are also functions of how many part 2 items one plans to order. As we show in the article, the optimal ordering decisions for both parts need to be made simultaneously in each period. The benefit of using such an integrated replenishment policy as opposed to two individual policies determined in isolation plus a coordinated effort in implementation, is substantial. Furthermore, errors caused by two individual policies obtained separately may not be compensated with coordination in the implementation phase. That is, the only way to capture all the significant cost saving opportunities is to make ordering decisions for both parts jointly in each period.

Two groups of literature are closely related to our research-perishable inventory systems and assembly systems. There has been considerable work on inventory control of perishable items and we refer readers to Nahmias [2] and Nahmias et al. [4] for a thorough review of papers in the area. However, the analysis of inventory systems with multiple products, some of which have perishable (fixed) lifetimes, is extremely difficult, and very few researchers have successfully identified the structure of the optimal policies. Nahmias and Pierskalla [3] derive the structure of the optimal policy for a two product system where one of the products has an infinite lifetime and the other one has a finite lifetime of $m$ periods. There is a single demand source which depletes the perishable stock first, according to the FIFO rule, and the nonperishable stock last. They show that there are exactly three ordering regions in each period which correspond to the three alternatives: ordering both products, ordering only perishable inventory, or not ordering. They characterize the region boundaries and the optimal policies for both the single period and the finite horizon dynamic problem. Our problem is different from Nahmias and Pierskalla's because, in our model, each final product requires one unit of the perishable item as well as one unit of the nonperishable item, whereas in their model demands are satisfied by either one unit of a perishable item or one unit of a nonperishable item.

There has been a body of work that focuses on the structure of the optimal policy in assembly systems. Among them, Rosling [5] considers a periodic review infinite horizon model of an assembly inventory system with random demands and proportional costs of production. Under a restriction on the initial stock levels and the absence of setup costs, he demonstrates that the assembly system can be remodeled as a series system. Schmidt and Nahmias [6] characterize the optimal ordering and assembly policies for a two-component assembly with random demand under a multiperiod horizon of arbitrary but finite length. A vast majority of the papers in this area assume no set up costs and none of them allows perishable components.

We provide a dynamic programming formulation of the problem with two state variables (on hand inventory of part 1 and backorders) and two decisions (the amount of part 1 to order and the amount of part 2 to order). We note that, even with two components, such a problem is quite challenging for the following reasons: (1) It allows both backorders and positive on hand inventory in each period as an order is fulfilled only when both items are available. (2) There are two decision variables representing the order-up-to levels for both parts. Because revenue is realized only after both items are put together, the cost of not fulfilling an order depends on the cause of stock-out (stock out of part 1 only, or part 2 only, or both) and decisions on quantities ordered for both parts need to be made simultaneously. (3) There exists a fixed ordering cost associated with one of the components. Although $K$-convexity has been a crucial ingredient for proving the optimality of an $(s, S)$ type policy, it is not obvious that such a property can be established with two state and two joint decision variables. As stated by Rosling [5], it is generally not possible to apply the series analogy, mentioned earlier, for assembly systems with no set-up costs to systems with setup costs, although his approach may be "a good approximation" for some special cases. For instance, his analysis may apply if one is allowed to order the nonperishable part in a fixed interval. This is the reason all research on assembly systems assumes away fixed ordering costs and most work focuses on performance evaluation under base-stock policies rather than structural analysis. We characterize the structure of the optimal policy by identifying the properties of the policy and the value function of the dynamic programming formulation. Most of the properties are proved using induction and some of the properties need to be proved concurrently in our induction.

We first analyze the optimal order quantity for part 2 for a given inventory level of part 1 after ordering. We show that the optimal order-up-to level of part 2 in a period is indeed the smaller of the inventory level of part 1 after ordering and a newsvendor type of solution that can be calculated in advance. The newsvendor type of solution depends on cost parameters associated with both part 1 and part 2 , but it is independent of the inventory level of part 1 after ordering. We incorporate this result into the dynamic program to determine the optimal order-up-to level of part 1 and, thereby, the optimal order-up-to level of part 2 that jointly minimize the expected discounted cost. We prove that the optimal order quantities for both parts in a period can be categorized as a function of the net inventory level of part 1 before ordering. When the net inventory level of part 1 is below a critical level, it is optimal to order part 1 to an optimal level obtained through 
joint optimization. Otherwise, no order of part 1 is necessary. The optimal order quantity for part 2 is either bounded by the optimal inventory level of part 1 or given by the newsvendor type of solution mentioned above. Our numerical study shows that the gain of using a joint replenishment policy is significant.

To the best of our knowledge, this work is the only exact characterization of an optimal policy for an assemblytype production system that includes both a perishable and nonperishable component, and a fixed ordering cost.

The remainder of this article is organized as follows. In Section 2, we introduce the dynamic programming formulation of the problem. In Section 3, we analyze the structure of the optimal policy by characterizing the optimal order quantities for part 1 and part 2. Having characterized the structure of the optimal policy, we present the results of a numerical study and demonstrate the benefits of using a joint replenishment policy in Section 4. Section 5 concludes the article with discussion on extensions.

\section{PROBLEM FORMULATION}

We consider a periodic review production system of two components, part 1 and part 2. Although the lifetime of part 1 is infinite, part 2 perishes after one period. An order (if any) for each part is placed at the beginning of each period, before observing the demand. We assume that a fixed ordering cost is incurred for part 1 only and replenishment orders arrive within the same period for both parts. Furthermore, the final product is assembed in response to demand and the capacity to assemble the two components to the final product is infinite. Demand for the final product occurs after an order (if any) is placed for each item and unmet demand is backordered. Our objective is to determine the quantity of each part to order for any given initial inventory of part 1 and backorder level so as to minimize the expected total discounted cost. We first introduce the following notation.

$K \quad$ fixed ordering cost for part 1

$c^{i} \quad$ purchasing cost per unit of part $i$

$\pi \quad$ backorder cost (lost of goodwill) per unit of demand

$h^{i} \quad$ holding cost per unit of part $i$

$P \quad$ selling price per unit $\left(P \geq c^{1}+c^{2}\right)$

$\beta \quad$ discount factor in period $n(\beta \leq 1)$

$\xi_{n} \quad$ demand in period $n$

$x_{n} \quad$ on hand inventory of part 1 at the beginning of period $n$ (state variable)

$w_{n}$ number of backorders at the beginning of period $n$ (state variable)

$y_{n}^{i} \quad$ order-up-to level for part $i$ after order delivery but before demand is realized and backorders are satisfied if any (decision variable)
We assume that $\xi_{n}$, where $n$ is the period index and read as "when there are $n$ periods to go," are independent and identically distributed positive random variables with probability density function $f$ and distribution function $F$. Although all cost parameters are assumed stationary, our results hold for problems with independent demands and nonstationary cost parameters under very general conditions.

Note that, even though part 2 is perishable, we include a holding cost $h^{2}$ for two reasons. First, holding costs may occur for the period in which part 2 is held, e.g., some chemicals need to be kept in controlled environment. Second, it can also be used to represent the cost for disposing excess perished units at the end of a period, if any.

For brevity, we write period $n$ instead of $n$ periods to go and drop the period subscript $n$ when we refer to the state $\left(x_{n}, w_{n}\right)$, both of which can be strictly positive, the decision variables $\left(y_{n}^{1}, y_{n}^{2}\right)$, and demand $\xi_{n}$. At the beginning of each period, one needs to decide the order-up-to levels for parts 1 and $2,\left(y^{1}, y^{2}\right)$, prior to demand realization and meeting backorders. Because any decision resulting in inventory level of part 2 higher than the inventory level of part 1 is strictly suboptimal, we only need to focus on the set of decisions with $y^{1} \geq y^{2}$. The objective is to determine the order-up-to levels $\left(y^{1}, y^{2}\right)$ that minimize the expected total discounted cost for any given state $(x, w)$. We say that order-up-to levels, $\left(y^{1}, y^{2}\right)$, are feasible in state $(x, w)$ if $y^{1} \geq x$ and $0 \leq y^{2} \leq y^{1}$ and define the set of feasible order-up-to levels at state $(x, w)$ in a period as $\mathcal{F}(x)=\left\{\left(y^{1}, y^{2}\right) \mid y^{1} \geq x\right.$ and $\left.0 \leq y^{2} \leq y^{1}\right\}$. Let $V_{n}(x, w)$ be the optimal expected discounted cost given that the current state is $(x, w)$ and there are $n$ periods to go for $n \geq 1$ and $V_{0}(\cdot, \cdot)=0$. We can then formulate the $n$-period problem, $n \geq 1$, using the following optimality equation.

$$
V_{n}(x, w)=\min _{\left(y^{1}, y^{2}\right) \in \mathcal{F}(x)}\left\{v_{n}\left(x, w, y^{1}, y^{2}\right)\right\}
$$

where

$$
\begin{gathered}
v_{n}\left(x, w, y^{1}, y^{2}\right)=K \delta\left(y^{1}-x\right)^{+}+c^{1}\left(y^{1}-x\right)+c^{2} y^{2} \\
+\int_{\max \left[0, y^{2}-w\right]}^{\infty}\left[\pi\left(z+w-y^{2}\right)+h^{1}\left(y^{1}-y^{2}\right)\right. \\
\left.-P y^{2}+\beta V_{n-1}\left(y^{1}-y^{2}, w+z-y^{2}\right)\right] f(z) d z \\
+\int_{0}^{\max \left[0, y^{2}-w\right]}\left[h^{1}\left(y^{1}-w-z\right)+h^{2}\left(y^{2}-w-z\right)\right. \\
\left.-P(z+w)+\beta V_{n-1}\left(y^{1}-z-w, 0\right)\right] f(z) d z
\end{gathered}
$$

is the expected discounted cost in state $(x, w)$ with $n$ periods to go for any feasible $\left(y^{1}, y^{2}\right)$ in period $n$ and when the optimal order-up-to levels are used in subsequent periods, and $\delta\left(y^{1}-x\right)^{+}=1$ if $y^{1}>x$ and 0 otherwise. 


\section{STRUCTURE OF THE OPTIMAL POLICY}

We now present a series of theorems which completely characterize the structure of the optimal policy. All proofs in the article can be found in the Appendix.

\section{THEOREM 1:}

(a) For any state $(x, w)$ and order-up-to level for part 1, $y^{1}$, the optimal order-up-to level for part 2 in period $n$ is $y^{2}=\min \left\{y^{1}, S_{n}^{2}+w\right\}$, where $S_{n}^{2}$ is the solution to

$$
F\left(S_{n}^{2}\right)= \begin{cases}\frac{\pi+P-c^{2}+h^{1}}{\pi+P+h^{1}+h^{2}}, & \text { if } n=1, \\ \frac{\pi+P-c^{2}+h^{1}-\beta\left(P-c^{2}\right)}{\pi+P+h^{1}+h^{2}-\beta\left(P-c^{2}\right)}, & \text { if } n \geq 2 .\end{cases}
$$

(b) $\left(y^{1}, y^{2}\right)$ is optimal in state $(x, w)$ if and only if $\left(y^{1}+\Delta, y^{2}+\Delta\right)$ is optimal in state $(x+\Delta, w+\Delta)$ for $\Delta \geq 0$. Furthermore, the difference of the expected costs, $V_{n}(x, w)-V_{n}(x+\Delta, w+\Delta)$, is equal to $\left(P-c^{2}\right) \Delta$.

Theorem 1 is critical and insightful in that, although the ordering decisions for both parts are coupled, $S_{n}^{2}$ itself is independent of the order-up-to level for part $1, y^{1}$, and the cost-to-go functions. One can obtain $S_{n}^{2}$ in advance by solving two independent newsvendor problems with costs associated with both parts 1 and 2. In each newsvendor problem, it is easy to see that the marginal overage cost is $c^{2}+h^{2}$. The costs associated with ordering one unit of part 2 less than the realized demand for $n \geq 2$ include the backorder cost, $\pi$, the holding cost for part $1, h^{1}$, the difference of the revenue between the current and the next period, $(1-\beta) P$, and the difference of the purchasing costs for part 2 between these same periods, $(\beta-1) c^{2}$. That is, the marginal underage cost is $\pi+P-c^{2}+h^{1}-\beta\left(P-c^{2}\right)$ for $n \geq 2$ and $\pi+P-c^{2}+h^{1}$ for $n=1$. Note that the purchasing cost of part $1, c^{1}$, does not affect $S_{n}^{2}$ as the cost for acquiring part 1 is a sunk cost.

Given $S_{n}^{2}$ for all $n$, the original dynamic programming problem is reduced to one with a single decision $y^{1}$ as $y^{2}=$ $\min \left\{y^{1}, S_{n}^{2}+w_{n}\right\}$. Therefore, the decision variable $y^{1}$ is determined by (1) the cost parameters associated with both parts and (2) the coordination constraints $y^{2}=\min \left\{y^{1}, S_{n}^{2}+w_{n}\right\}$ in subsequent periods. If we view $y^{1}$ as the supplier capacity for part 2, the ordering decision for part 2 is the same as that in the capacitated stochastic inventory system with no fixed ordering cost (see Ref. [1]), except that $y^{2}$ is bounded by a state-dependent inventory level for part $1, y^{1}$, rather than an exogenous capacity limit.

Theorem 1, together with Lemmas 1-3 in the Appendix, help us prove Theorem 2 which is critical for identifying the optimal order-up-to level for part 1.

THEOREM 2: If it is optimal to order part 1 in state $(x, w)$ and the optimal inventory levels for that state are $\left(y^{1}, y^{2}\right)$, then it is also optimal to order part 1 in state $(x, w+\Delta)$ for $\Delta \geq 0$ and the corresponding optimal inventory levels are $\left(y^{1}+\Delta, y^{2}+\Delta\right)$. Furthermore, the difference of the expected costs, $V_{n}(x, w)-V_{n}(x, w+\Delta)$, is linear in $\Delta$ and equal to $\left(P-c^{1}-c^{2}\right) \Delta$.

The proof of Theorem 2 is fairly involved. We need to prove the following properties in each step of our induction on the period $n$ concurrently and the theorem follows directly from the last two properties.

(a) If it is optimal to order part 1 in state $(x, w)$ (i.e., $\left.y^{1}>x\right)$ and the optimal order-up-to levels are $\left(y^{1}, y^{2}\right)$, then $y^{2}>x$ and $y^{2} \geq w$.

(b) If it is optimal to order part 1 in state $(x, w)$, then it is also optimal to order part 1 in state $(x, w+\Delta)$ for $\Delta \geq 0$.

(c) If it is optimal to order part 1 in state $(x, w)$ and the optimal order-up-to levels are $\left(y^{1}, y^{2}\right)$, then the optimal order-up-to levels for state $(x, w+\Delta), \Delta \geq 0$, is $\left(y^{1}+\Delta, y^{2}+\Delta\right)$ and $V_{n}(x, w)-V_{n}(x, w+\Delta)=$ $\left(P-c^{1}-c^{2}\right) \Delta$.

Theorem 2 indicates that there exists a threshold $\hat{w}_{x}$ for any $x$ such that it is optimal to order part 1 if $w \geq \hat{w}_{x}$ and the order quantities increase by $w-\hat{w}_{x}$. No order of part 1 should be placed if $w<\hat{w}_{x}$. By Theorem 1(b), this threshold increases by exactly the amount of increase in $x$, i.e., $\hat{w}_{x+\Delta}=\hat{w}_{x}+\Delta$. Therefore, $(x+\Delta)-\hat{w}_{x+\Delta}=x+\Delta-\left(\hat{w}_{x}+\Delta\right)=x-\hat{w}_{x}$ and the decision on when to order at $(x, w)$ can be completely determined by comparing the net inventory level, $x-w$, to a constant threshold value $s_{n}=x-\hat{w}_{x}$ described in Theorem 3 .

THEOREM 3: In any period $n$ and state $(x, w)$, it is optimal to order part 1 if and only if $x-w$ is less than or equal to a constant $s_{n}$.

Although Theorem 3 ensures the existence of an optimal inventory ordering level for part 1 , the next theorem shows that, in states where it is optimal to order part 1 , one always orders enough to bring its net inventory up to the same level. Note that the order-up-to levels can be different in different periods in a finite horizon problem.

THEOREM 4: In any period $n$ and state $(x, w)$, if it is optimal to order part 1, then the amount ordered will always bring the net inventory $x-w$ to an optimal order-up-to level, $S_{n}^{1}$, which is a constant. That is, one always raises the inventory level for part 1 to $S_{n}^{1}+w$.

The proof follows directly from part (b) of Theorem 1 and Theorem 3. Combining Theorems 3 and 4, we have demonstrated the existence of a threshold $s_{n}$ such that it is optimal to 


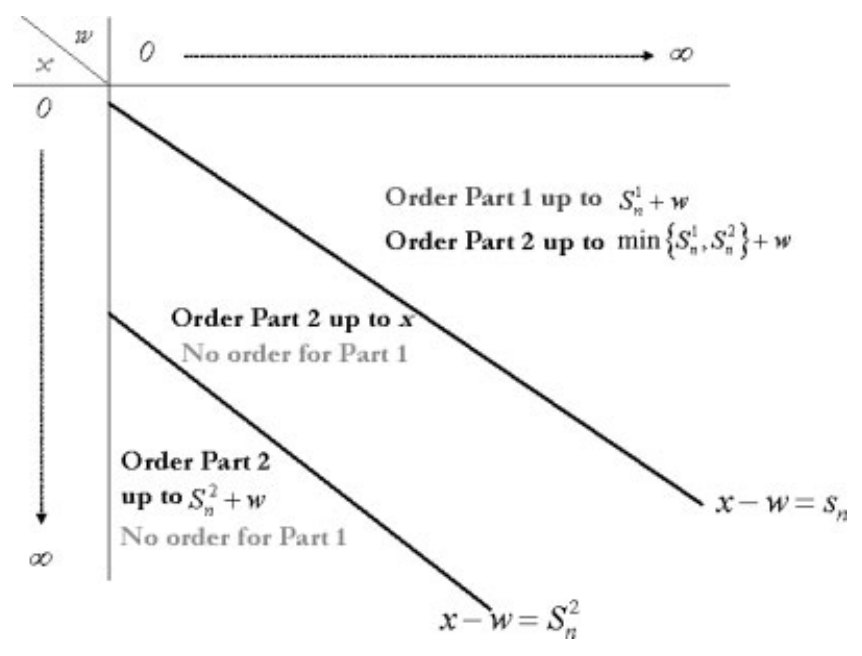

Figure 1. The structure of the optimal policy.

order part 1 whenever the net inventory $x-w \leq s_{n}$ and that, whenever it is optimal to order part 1 , it is optimal to order up to $S_{n}^{1}+w$. Given the decision on part 1's inventory, it is optimal to order part 2 up to $S_{n}^{2}+w$ if the amount available of part 1 is at least $S_{n}^{2}+w$. Otherwise, it is optimal to order part 2 up to the amount of part 1 available.

By Theorems 1 through 4, we now characterize the structure of the optimal policy.

THEOREM 5: There exist values $\left(s_{n}, S_{n}^{1}, S_{n}^{2}\right)$ such that it is optimal to order part 1 if $x-w \leq s_{n}$. When it is optimal to order part 1 , one always orders $S_{n}^{1}-x+w$ units to raise the inventory of part 1 to $y^{1}=S_{n}^{1}+w$. For part 2 , it is optimal to bring the inventory level up to $y^{2}=\min \left\{y^{1}, S_{n}^{2}+w\right\}$.

On the basis of Theorem 5, the optimal order quantities fall into one of the three regions at any state $(x, w)$ shown in Fig. 1. (i) Order part 1 up to $S_{n}^{1}+w$ and part 2 up to $\min \left\{S_{n}^{1}, S_{n}^{2}\right\}+w$, (ii) order only part 2 up to $x$, and (iii) order only part 2 up to $S_{n}^{2}+w$. That is, when the net inventory level for the nonperishable part is too low (i.e., $x-w \leq s_{n}$ ) and an order of part 1 is triggered, one raises the inventory level of part 1 to a sufficiently high level, $S_{n}^{1}+w$, and the inventory level for part 2 to $\min \left\{S_{n}^{1}, S_{n}^{2}\right\}+w$. On the other hand, when the net inventory level for the nonperishable part is neither sufficiently high nor low (i.e., $s_{n}<x-w \leq S_{n}^{2}$ ), it is optimal not to order part 1 and the optimal order-up-to level for the perishable part is bounded by the available inventory level of part $1, x$. Finally, when the net inventory level for the nonperishable part is sufficiently high (i.e., $x-w>S_{n}^{2}$ ) and no order is placed for part 1 , the "non-capacitated" order-up-to level of the perishable part, $S_{n}^{2}+w$, is realized.

\section{NUMERICAL STUDY}

As we have shown in the previous section, the optimal decisions for both parts $\left(y^{1}, y^{2}\right)$ determined by $\left(s_{n}, S_{n}^{1}, S_{n}^{2}\right)$ are not only coupled, but also state dependent. We now compare the optimal policy with a heuristic which has the same structure as the optimal policy except that $\left(s_{n}, S_{n}^{1}\right)$ and $S_{n}^{2}$ are determined by solving two individual inventory problems in isolation. That is, one determines $S_{n}^{2}$ by solving a newsvendor problem with underage cost $\pi+P-c^{2}-\beta\left(P-c^{2}\right)$ for $n \geq 2\left(\pi+P-c^{2}\right.$ for $\left.n=1\right)$ and overage cost $c^{2}+h^{2}$, and obtains $\left(s_{n}, S_{n}^{1}\right)$ by solving a single item inventory problem with parameters $\pi, P, K, c^{1}$, and $h^{1}$ and ignoring the coordination constraint. Once these parameters are determined, one then coordinates the replenishment of the inventory for the two parts by setting $y^{2}=\min \left\{y^{1}, S_{n}^{2}+w\right\}$ only in the implementation phase. We label this heuristic as Coordinated.

We constructed examples that cover a wide range of scenarios and used both discrete uniform and triangular demand distributions given the programming complexity of continuous demand functions. To compare different policies free of the end-of-horizon effect, we focused on the infinite horizon versions on a truncated state space and (hence) finite action space. Thus, the solution of the infinite horizon program exists. We fixed the discount rate at $\beta=0.95$ and the holding cost for part 2 at $h^{2}=0.25$ and varied other parameters. We considered $K=50,150, P=8,16, \pi=3,9$, $c^{1}=1,2, c^{2}=1,2, h^{1}=0.25,0.5$, a discrete uniform distribution $U[0,9]$ and a discrete triangular distribution $T[0,9]$ where

$\left(p_{0}, p_{1}, p_{2}, p_{3}, p_{4}, p_{5}, p_{6}, p_{7}, p_{8}, p_{9}\right)=(0.025,0.0375$,

$0.0625,0.125,0.25,0.25,0.125,0.0625,0.0375,0.025)$.

For each combination of $K, P$, and $\pi$ values, and demand distributions, we computed both the optimal policies and heuristic policies for the following six examples:

$\left(c^{1}, c^{2}, h^{1}\right) \in\{(2,2, .025),(2,2,0.5),(1,2,0.25),(1,2,0.5)$,

$(2,1,0.25),(2,1,0.5)\}$.

In Table 1, we reported the average, maximum, and minimum percentage suboptimality of the expected profits (rather than the expected costs as our cost function includes the revenue) for using the heuristic policy when demand follows the uniform distribution. The results for the triangular distribution are qualitatively similar, therefore omitted. As we can see, the heuristic policy performs poorly in most cases. In some cases, the heuristic policy results in losses whereas the profits under the optimal policy are strictly positive (i.e., the percentage suboptimality is greater than $100 \%$ ). In the same table, we also reported the performance of a heuristic under 
Table 1. Comparisons of profits and policies for $\beta=0.95, h^{2}=0.25$, and $\xi \sim U[0,9]$.

\begin{tabular}{|c|c|c|c|c|c|c|c|}
\hline \multirow[b]{2}{*}{ No. } & \multirow[b]{2}{*}{$K$} & \multirow[b]{2}{*}{$P$} & \multirow[b]{2}{*}{$\pi$} & \multicolumn{2}{|c|}{ Independent (\% Suboptimality) } & \multicolumn{2}{|c|}{ Coordinated (\% Suboptimality) } \\
\hline & & & & Average & (Max, Min) & Average & (Max, Min) \\
\hline 1 & 50 & 8 & 3 & 28.7 & $(41.7,12.7)$ & 21.7 & $(31.1,11)$ \\
\hline 2 & 50 & 8 & 9 & 191.8 & $(821.3,21.3)$ & 144.1 & $(610.4,14.5)$ \\
\hline 3 & 50 & 16 & 3 & 8.0 & $(10.7,4.6)$ & 5.8 & $(7.8,3.7)$ \\
\hline 4 & 50 & 16 & 9 & 7.3 & $(11.9,4.2)$ & 5.4 & $(8.7,2.8)$ \\
\hline 5 & 150 & 8 & 3 & 140.0 & $(251.7,73.5)$ & 113.8 & $(209.1,64.3)$ \\
\hline 6 & 150 & 8 & 9 & 199.2 & $(684,74.6)$ & 150.3 & $(508.2,56)$ \\
\hline 7 & 150 & 16 & 3 & 18.8 & $(25,11.9)$ & 15.1 & $(20.1,10.2)$ \\
\hline 8 & 150 & 16 & 9 & 15.8 & $(25.8,9.1)$ & 12.0 & $(19.1,7.2)$ \\
\hline
\end{tabular}

which one further neglects to coordinate the replenishment during implementation, labeled as the Independent heuristic. Intuitively, coordination reduces overstock of either part and improves the performance. The difference between the profits under the two heuristics represents the value of coordination in the implementation phase. It turned out that coordination does not improve the performance significantly in most of the examples, indicating the importance of obtaining the optimal $\left(s, S^{1}, S^{2}\right)$ in the first place.

The benefits of using an optimal policy are most significant in cases with high costs and/or low profit margins (e.g., lower selling prices and/or higher operating and material costs). These can be seen in additional examples in Tables 2 and 3 in which we compare the profits and policies as functions of $K$ and $c^{2}$. The results for other parameters are similar and hence are not reported here. Table 2 shows that the benefits of using an optimal policy over a coordinated policy increase as $K$ increases. For the unit cost of part 2, Table 3 indicates that the percentage increase in benefits has an increasing trend, but it is not always monotone.

In general, the heuristic returns values of $\left(s, S^{1}\right)$ and $S^{2}$ that are at least as high as, in most cases significantly higher than, those in an optimal solution due to the way the profit margin and stockout costs are used in the calculations. The extra inventory costs cause the profit gap between the optimal policy and the coordinated heuristic policy. It is important to note that the heuristic leads to significantly higher $\left(s, S^{1}\right)$ values, even when $S^{2}$ happens to be the optimal as in examples No. 9, 10, 12, 21, and 24 in Table 3.

\section{CONCLUSIONS AND FURTHER RESEARCH}

In this article, we consider a periodic review make-to-order system where one nonperishable part, part 1, and one perishable part, part 2, are needed to fulfill a customer order. Assuming that there is a fixed ordering cost associated with part 1 and all customers are willing to wait, we show that the optimal policy is guided by an $(s, S)$ policy for part 1 and a modified base stock policy for part 2. The parameters $s$ and $S$ depend on the costs associated with part 2 and the coordination constraint, while the base-stock level for part 2 explicitly depends on the inventory level of part 1, the backorder level, and the costs associated with part 1 .

Through extensive numerical study, we demonstrated that the additional profits realized by a joint replenishment policy for both parts can be significant relative to the policy that considers the inventory policy for each part separately. In general, the joint inventory policy is most valuable in systems with high costs and/or low profit margins.

Table 2. Comparisons of profits and policies as functions of $K$.

\begin{tabular}{|c|c|c|c|c|c|c|c|}
\hline \multirow[b]{2}{*}{ No. } & \multirow[b]{2}{*}{$K$} & \multicolumn{2}{|c|}{ Optimal policy } & \multicolumn{4}{|c|}{ Coordinated } \\
\hline & & $\left(s, S^{1}, S^{2}\right)$ & Profit & $\left(s, S^{1}\right)$ & $S^{2}$ & Profit & $\%$ Opt. \\
\hline 1 & 5 & $(5,12,7)$ & 910.83 & $(8,15)$ & 8 & 873.47 & 4.1 \\
\hline 2 & 10 & $(5,14,7)$ & 886.5 & $(8,16)$ & 8 & 843.40 & 4.9 \\
\hline 3 & 20 & $(5,18,7)$ & 850.07 & $(7,22)$ & 8 & 806.70 & 5.1 \\
\hline 4 & 50 & $(3,28,7)$ & 773.14 & $(6,31)$ & 8 & 718.54 & 7.1 \\
\hline 5 & 75 & $(3,28,7)$ & 721.75 & $(5,36)$ & 8 & 662.46 & 8.2 \\
\hline 6 & 100 & $(3,31,7)$ & 675.93 & $(5,40)$ & 8 & 607.00 & 10.2 \\
\hline 7 & 150 & $(4,36,7)$ & 594.18 & $(4,47)$ & 8 & 513.35 & 13.6 \\
\hline 8 & 200 & $(2,39,7)$ & 520.65 & $(4,47)$ & 8 & 429.77 & 17.5 \\
\hline 9 & 300 & $(1,44,7)$ & 387.56 & $(2,61)$ & 8 & 272.38 & 29.7 \\
\hline 10 & 500 & $(0,47,7)$ & 267.97 & $(1,74)$ & 8 & -11.95 & 104.5 \\
\hline
\end{tabular}

Parameters: $\beta=0.95, P=16, \pi=9, c^{1}=c^{2}=2, h^{1}=h^{2}=0.25$, and $\xi \sim U[0,9]$. 
Table 3. Comparisons of profits and policies as functions of $K$ and $c^{2}$.

\begin{tabular}{|c|c|c|c|c|c|c|c|c|}
\hline \multirow[b]{2}{*}{ No. } & \multirow[b]{2}{*}{$K$} & \multirow[b]{2}{*}{$c^{2}$} & \multicolumn{2}{|c|}{ Optimal } & \multicolumn{4}{|c|}{ Coordinated } \\
\hline & & & $\left(s, S^{1}, S^{2}\right)$ & Profit & $\left(s, S^{1}\right)$ & $S^{2}$ & Profit & $\%$ Opt. \\
\hline 1 & 50 & 0.5 & $(4,27,8)$ & 979.70 & $(5,34)$ & 9 & 924.88 & 5.6 \\
\hline 2 & 50 & 0.75 & $(4,26,7)$ & 944.39 & $(5,34)$ & 9 & 881.08 & 6.7 \\
\hline 3 & 50 & 1 & $(4,26,7)$ & 909.40 & $(5,31)$ & 8 & 883.98 & 2.8 \\
\hline 4 & 50 & 1.25 & $(4,26,7)$ & 874.65 & $(6,31)$ & 8 & 838.98 & 4.1 \\
\hline 5 & 50 & 1.5 & $(4,25,7)$ & 840.46 & $(6,31)$ & 8 & 798.84 & 5.0 \\
\hline 6 & 50 & 1.75 & $(4,25,7)$ & 806.59 & $(6,31)$ & 8 & 758.69 & 5.9 \\
\hline 7 & 50 & 2 & $(4,24,7)$ & 773.13 & $(6,31)$ & 8 & 718.54 & 7.1 \\
\hline 8 & 50 & 3 & $(3,23,6)$ & 643.62 & $(6,28)$ & 7 & 610.32 & 5.2 \\
\hline 9 & 50 & 4 & $(3,22,6)$ & 518.47 & $(6,25)$ & 6 & 499.27 & 3.7 \\
\hline 10 & 50 & 5 & $(3,21,6)$ & 398.40 & $(6,25)$ & 6 & 367.87 & 7.7 \\
\hline 11 & 50 & 6 & $(3,20,5)$ & 282.25 & $(6,25)$ & 6 & 236.47 & 16.2 \\
\hline 12 & 50 & 8 & $(2,29,5)$ & 57.42 & $(7,24)$ & 5 & 16.12 & 71.9 \\
\hline 13 & 150 & 0.5 & $(3,39,8)$ & 792.61 & $(3,52)$ & 9 & 703.02 & 11.3 \\
\hline 14 & 150 & 0.75 & $(3,38,7)$ & 758.69 & $(3,52)$ & 9 & 659.44 & 13.1 \\
\hline 15 & 150 & 1 & $(3,38,7)$ & 725.14 & $(4,47)$ & 8 & 672.93 & 7.2 \\
\hline 16 & 150 & 1.25 & $(3,37,7)$ & 691.83 & $(4,47)$ & 8 & 633.03 & 8.5 \\
\hline 17 & 150 & 1.5 & $(2,37,7)$ & 658.92 & $(4,47)$ & 8 & 593.14 & 10.0 \\
\hline 18 & 150 & 1.75 & $(2,36,7)$ & 626.33 & $(4,47)$ & 8 & 553.24 & 11.7 \\
\hline 19 & 150 & 2 & $(2,36,7)$ & 594.18 & $(4,47)$ & 8 & 513.35 & 13.6 \\
\hline 20 & 150 & 3 & $(2,34,6)$ & 499.45 & $(4,42)$ & 7 & 418.53 & 16.2 \\
\hline 21 & 150 & 4 & $(2,32,6)$ & 348.52 & $(5,38)$ & 6 & 314.81 & 9.7 \\
\hline 22 & 150 & 5 & $(2,30,5)$ & 231.76 & $(5,38)$ & 6 & 183.61 & 20.8 \\
\hline 23 & 150 & 6 & $(1,29,5)$ & 118.89 & $(5,38)$ & 6 & 52.41 & 55.9 \\
\hline 24 & 150 & 8 & $(1,27,5)$ & -99.06 & $(5,34)$ & 5 & -149.67 & 51.1 \\
\hline
\end{tabular}

Parameters: $\beta=0.95, P=16, \pi=9, c^{1}=2, h^{1}=h^{2}=0.25$, and $\xi \sim U[0,9]$.

Further research should address more complicated situations as described below.

- More than two items: Our analysis can be easily extended to multiple perishable parts, each with a one period life, with no fixed ordering costs. Because the order up to levels for all perishable parts should be the same and there is no fixed ordering cost associated with these parts, we can group these parts into a kit as part 2 and all results hold. However, incorporating multiple nonperishable parts is more difficult, especially with fixed ordering costs. In practice, one may either focus on one major nonperishable component or bundle several nonperishable parts into one subassembly while making inventory decisions.

- Lost sales: With lost sales instead of backorders, $w=0$ in all periods and there is only one state variable $x$, representing the net inventory level for part 1. Our preliminary research indicates that the optimal structure is very similar, except that the net order-upto level of part 2, $S_{n}^{2}$, is no longer independent of the stock level of part 1 and the current state. That is, $y^{2}$ depends on $y^{1}$ in a more complicated fashion and Theorem 1 no longer holds.

- Make-to-stock environment: The optimal policy under a make-to-stock system depends on the shelf-life of the final product and the inventory holding cost of the final product which may not be the sum of the holding costs for both components. For example, if the final product is highly perishable and lasts only one period, then it is not difficult to show that our result also holds. On the other hand, if the final product has a very long or infinite shelf-life and the inventory holding cost of the final product is not too high (such as canned food), then it is very likely that a policy that orders the same quantity for both parts and only keeps the inventory of final product is optimal. In such case, the problem can be simplified to a singleitem inventory problem with a unit cost $c_{n}=c^{1}+c^{2}$ and a fixed ordering cost $K$, thus an $(s, S)$ policy is optimal. With the exception of these special cases, the optimal policy is quite complex. That is, it can be state dependent and depends on the shelf-life of the final product. Therefore, the method in this paper is not likely to work.

- Positive lead times: We expect that a positive order lead time for part 1 would not affect the structure of the optimal policy. However, if there is a positive lead time for part 2, it is not clear how the optimal policy for part 2 behaves. Ideally, we would not like to receive more part 2 than the amount of part 1 available when an order arrives. However, the amount of part 1 will 
be available when an order of part 2 arrives would be unknown when part 2 items are ordered. Therefore, the order-up-to level for part 2 depends on the current inventory and backorder levels as well as the distribution of demand during the lead time. For instance, it is evident that a policy that delivers fewer units of part 2 when the projected inventory level of part 1 is low will outperform a policy that orders up to a constant stock level. On the other hand, the optimal order-up-to quantity could be equal to a constant level $S_{n}^{2}$ plus $w$ when the projected inventory level of part 1 is high. We conjecture that the optimal policy for part 2 in this case is the minimum of $S_{n}^{2}+w$ and $E\left(y^{1}(\tau)\right)$, the "expected" quantity of part 1 when the order for part 2 arrives after a positive lead time, but we are not yet able to prove this. We are also unable to fully identify the form of $E\left(y^{1}(\tau)\right)$, although we know it depends on $x$ and $w$.

- A longer lifetime of part 2: With a longer lifetime, the problem becomes much more complicated, since the state vector must include the stock level of each possible age category. If the lifetime of part 2 were $m$ periods, the state vector would be $\left(x^{1}, x_{1}^{2}, x_{2}^{2}, \ldots, x_{m}^{2}, w\right)$, where $x^{1}$ is the stock level of part 1 and $x_{k}^{2}$ is the stock level of part 2 with age $k$. These problems can be formulated and solved using dynamic programming, but characterizing the structure of the optimal policy is a formidable task in the presence of a fixed ordering cost for part 1 .

Although some of the above mentioned extensions are challenging, we believe that the insight obtained from our work will shed light on developing efficient solution approaches to these extensions.

\section{APPENDIX}

PROOF OF THEOREM 1: We use induction on the period, $n$, to prove these two properties concurrently.

\section{For period 1:}

(a) To obtain the optimal inventory level of part 2 for a given $y^{1}$ large enough, we first consider the case when $y^{2}>w$ and take the derivative of $v_{1}\left(x, w, y^{1}, y^{2}\right)$ with respect to $y^{2}$ :

$$
\begin{gathered}
v_{1}\left(x, w, y^{1}, y^{2}\right)=K \delta\left(y^{1}-x\right)^{+}+c^{1}\left(y^{1}-x\right)+c^{2} y^{2} \\
+\int_{y^{2}-w}^{\infty}\left[\pi\left(z+w-y^{2}\right)+h^{1}\left(y^{1}-y^{2}\right)-P y^{2}\right] f(z) d z \\
+\int_{0}^{y^{2}-w}\left[h^{1}\left(y^{1}-w-z\right)+h^{2}\left(y^{2}-w-z\right)-P(z+w)\right] f(z) d z, \\
\frac{\partial v_{1}\left(x, w, y^{1}, y^{2}\right)}{\partial y^{2}}=c^{2}-\left(\pi+h^{1}+P\right) \int_{y^{2}-w}^{\infty} f(z) d z+h^{2} \int_{0}^{y^{2}-w} f(z) d z \\
=c^{2}-\left(\pi+h^{1}+P\right)\left[1-F\left(y^{2}-w\right)\right]+h^{2} F\left(y^{2}-w\right) .
\end{gathered}
$$

The solution of the first-order condition is obtained at $S_{1}^{2}$, which satisfies

$$
F\left(y^{2}-w\right)=F\left(S_{1}^{2}\right)=\frac{\pi+P-c^{2}+h^{1}}{\pi+P+h^{1}+h^{2}} .
$$

The existence of solution follows immediately from the fact that $P>c^{2}$. To show this $S_{1}^{2}$ is indeed optimal, we show the convexity of the cost function by taking the second derivative of $v_{1}\left(x, w, y^{1}, y^{2}\right)$ with respect to $y^{2}$,

$$
\frac{\partial^{2} v_{1}\left(x, w, y^{1}, y^{2}\right)}{\partial\left(y^{2}\right)^{2}}=\left(\pi+P+h^{1}+h^{2}\right) f\left(y^{2}-w\right)>0 .
$$

Therefore, $S_{1}^{2}$ minimizes the cost. Applying similar algebra, we obtain the cost function and its derivative for $y^{2} \leq w$ :

$$
\begin{gathered}
v_{1}\left(x, w, y^{1}, y^{2}\right)=K \delta\left(y^{1}-x\right)^{+}+c^{1}\left(y^{1}-x\right)+c^{2} y^{2} \\
+\int_{0}^{\infty}\left[\pi\left(z+w-y^{2}\right)+h^{1}\left(y^{1}-y^{2}\right)-P y^{2}\right] f(z) d z, \\
\frac{\partial v_{1}\left(x, w, y^{1}, y^{2}\right)}{\partial y^{2}}=c^{2}-\left(\pi+h^{1}+P\right)<0 .
\end{gathered}
$$

The fact that the cost function is convex for $y^{2}>w$ and the fact that $v_{1}\left(x, w, y^{1}, y^{2}\right)$ is strictly decreasing in $y^{2}$ for $y^{2} \leq w$ implies that it is optimal to order the maximum feasible quantity, $y^{1}$, when $y^{1}<S_{1}^{2}+w$. Therefore, $y^{2}=\min \left\{y^{1}, S_{1}^{2}+w\right\}$.

(b) To help the reader understand this approach, we first sketch the steps of the proof:

(i) For any feasible pair of order-up-to levels $\left(y^{1}, y^{2}\right)$ in state $(x, w)$, we prove that for any $\Delta>0, v_{1}(x+\Delta$, $\left.w+\Delta, y^{1}+\Delta, y^{2}+\Delta\right)=V_{1}(x, w)+\left(c^{2}-P\right) \Delta$ and hence, $V_{1}(x+\Delta, w+\Delta) \leq V_{1}(x, w)+\left(c^{2}-P\right) \Delta$.

(ii) Similarly, we prove the inequality in the opposite direction. We show that if $\left(y^{1}, y^{2}\right)$ is optimal in state $(x+\Delta, w+\Delta)$, then $v_{1}\left(x, w, y^{1}-\Delta, y^{2}-\Delta\right)=V_{1}(x+\Delta, w+\Delta)-\left(c^{2}-P\right) \Delta$ and $V_{1}(x+\Delta, w+\Delta) \geq V_{1}(x, w)+\left(c^{2}-P\right) \Delta$.

By (i) and (ii), $V_{1}(x+\Delta, w+\Delta)=V_{1}(x, w)+\left(c^{2}-P\right) \Delta=$ $v_{1}\left(x+\Delta, w+\Delta, y^{1}+\Delta, y^{2}+\Delta\right)$ and $\left(y^{1}+\Delta, y^{2}+\Delta\right)$ is optimal in state $(x+\Delta, w+\Delta)$.

Suppose that $\left(y^{1}, y^{2}\right)$ is optimal in state $(x, w)$. A simple inspection shows that raising inventories levels of both components to $\left(y^{1}+\Delta, y^{2}+\Delta\right)$ must be feasible in state $(x+\Delta, w+\Delta)$. Because $\Delta$ additional units of the end item are produced and sold in the same period and the Markov chain induced by $\left(y^{1}+\Delta, y^{2}+\Delta\right)$ will result in the same state as the one induced by $\left(y^{1}, y^{2}\right)$ in state $(x, w)$ for any realization of demand. For $y^{2}>w$, the cost associated with raising inventory levels to $\left(y^{1}+\Delta, y^{2}+\Delta\right)$ in state $(x+\Delta, w+\Delta)$ is:

$$
\begin{aligned}
& v_{1}\left(x+\Delta, w+\Delta, y^{1}+\Delta, y^{2}+\Delta\right) \\
& =K \delta\left(y^{1}-x\right)^{+}+c^{1}\left(y^{1}-x\right)+c^{2}\left(y^{2}+\Delta\right) \\
& +\int_{y^{2}-w}^{\infty}\left[\pi\left(z+w-y^{2}\right)+h^{1}\left(y^{1}-y^{2}\right)-P\left(y^{2}+\Delta\right)\right] f(z) d z \\
& +\int_{0}^{y^{2}-w}\left[h^{1}\left(y^{1}-w-z\right)+h^{2}\left(y^{2}-w-z\right)-P(z+w+\Delta)\right] f(z) d z \\
& =V_{1}(x, w)+\left(c^{2}-P\right) \Delta .
\end{aligned}
$$


Similarly, for $y^{2} \leq w$, the cost associated with $\left(y^{1}+\Delta, y^{2}+\Delta\right)$ is:

$$
\begin{array}{r}
v_{1}\left(x+\Delta, w+\Delta, y^{1}+\Delta, y^{2}+\Delta\right) \\
=K \delta\left(y^{1}-x\right)^{+}+c^{1}\left(y^{1}-x\right)+c^{2}\left(y^{2}+\Delta\right) \\
+\int_{0}^{\infty}\left[\pi\left(z+w-y^{2}\right)+h^{1}\left(y^{1}-y^{2}\right)-P\left(y^{2}+\Delta\right)\right] f(z) d z \\
=V_{1}(x, w)+\left(c^{2}-P\right) \Delta .
\end{array}
$$

Therefore, $V_{1}(x+\Delta, w+\Delta) \leq V_{1}(x, w)+\left(c^{2}-P\right) \Delta$.

To show the inequality holds for the opposite direction, we first note that, if $\left(y^{1}, y^{2}\right)$ is optimal in state $(x+\Delta, w+\Delta)$, then $\left(y^{1}-\Delta, y^{2}-\Delta\right)$ must be feasible in state $(x, w)$. This is because $y^{1} \geq x+\Delta$. Furthermore, as we already proved in the part (a) of Theorem 1 that $y^{2}=\min \left\{y^{1}, S_{1}^{2}+w+\Delta\right\}$ for $(x+\Delta, w+\Delta)$, $y^{2}-\Delta>0$. Hence, $\left(y^{1}-\Delta, y^{2}-\Delta\right)$ is feasible in state $(x, w)$. Implementing $\left(y^{1}-\Delta, y^{2}-\Delta\right)$ in state $(x, w)$ will yield the following expected cost for $y^{2}-\Delta \geq w$ :

$$
\begin{array}{r}
v_{1}\left(x, w, y^{1}-\Delta, y^{2}-\Delta\right)=K \delta\left(y^{1}-\Delta-x\right)^{+}+c^{1}\left(y^{1}-\Delta-x\right) \\
+c^{2}\left(y^{2}-\Delta\right)+\int_{y^{2}-\Delta-w}^{\infty}\left[\pi\left(z+w-y^{2}+\Delta\right)+h^{1}\left(y^{1}-y^{2}\right)\right. \\
\left.-P\left(y^{2}-\Delta\right)\right] f(z) d z+\int_{0}^{y^{2}-\Delta-w}\left[h^{1}\left(y^{1}-\Delta-w-z\right)\right. \\
\left.+h^{2}\left(y^{2}-\Delta-w-z\right)-P(z+w)\right] f(z) d z \\
=V_{1}(x+\Delta, w+\Delta)-\left(c^{2}-P\right) \Delta,
\end{array}
$$

and we have $V_{1}(x, w) \leq V_{1}(x+\Delta, w+\Delta)-\left(c^{2}-P\right) \Delta$. If $y^{2}-\Delta<w$, then the second integral disappears but the result holds.

Combining two inequalities, we conclude that $V_{1}(x, w)=$ $V_{1}(x+\Delta, w+\Delta)-\left(c^{2}-P\right) \Delta$ and the optimal order-up-to levels for both parts in state $(x+\Delta, w+\Delta)$ are $\left(y^{1}+\Delta, y^{2}+\Delta\right)$.

For period $n-1$ : Assume

(a) $y^{2}=\min \left\{y^{1}, S_{n-1}^{2}+w\right\}$, where

$$
F\left(S_{n-1}^{2}\right)= \begin{cases}\frac{\pi+P-c^{2}+h^{1}}{\pi+P+h^{1}+h^{2}}, & \text { if } n=2, \\ \frac{\pi+P-c^{2}+h^{1}-\beta\left(P-c^{2}\right)}{\pi+P+h^{1}+h^{2}-\beta\left(P-c^{2}\right)}, & \text { if } n \geq 3 .\end{cases}
$$

(b) $\left(y^{1}, y^{2}\right)$ is optimal in state $(x, w)$, if and only if $\left(y^{1}+\Delta, y^{2}+\Delta\right)$ is optimal in state $(x+\Delta, w+\Delta)$. Moreover, $V_{n-1}(x, w)-V_{n-1}(x+$ $\Delta, w+\Delta)=\left(P-c^{2}\right) \Delta$.

For period $n$ : We present the proof for $n \geq 3$ as the proof for $n=2$ follows identical steps.

(a) The cost under a policy that orders $\left(y^{1}, y^{2}\right)$ in period $n$ and follows the optimal policy afterward, $v_{n}\left(x, w, y^{1}, y^{2}\right)$ is

$$
\begin{gathered}
v_{n}\left(x, w, y^{1}, y^{2}\right)=K \delta\left(y^{1}-x\right)^{+}+c^{1}\left(y^{1}-x\right)+c^{2} y^{2} \\
+\int_{y^{2}-w}^{\infty}\left[\pi\left(z+w-y^{2}\right)+h^{1}\left(y^{1}-y^{2}\right)-P y^{2}\right. \\
\left.+\beta V_{n-1}\left(y^{1}-y^{2}, w+z-y^{2}\right)\right] f(z) d z \\
+\int_{0}^{y^{2}-w}\left[h^{1}\left(y^{1}-w-z\right)+h^{2}\left(y^{2}-w-z\right)\right. \\
\left.\quad-P(z+w)+\beta V_{n-1}\left(y^{1}-z-w, 0\right)\right] f(z) d z,
\end{gathered}
$$

and its first-order condition with respect to $y^{2}$ is

$$
\begin{array}{r}
\frac{\partial v_{n}\left(x, w, y^{1}, y^{2}\right)}{\partial y^{2}}=c^{2}-\left(\pi+h^{1}+P\right) \int_{y^{2}-w}^{\infty} f(z) d z \\
+\beta \int_{y^{2}-w}^{\infty} \frac{\partial V_{n-1}\left(y^{1}-y^{2}, w+z-y^{2}\right)}{\partial y^{2}} f(z) d z \\
\quad+h^{2} \int_{0}^{y^{2}-w} f(z) d z \\
=c^{2}-\left(\pi+h^{1}+P\right)\left[1-F\left(y^{2}-w\right)\right] \\
\quad+h^{2} F\left(y^{2}-w\right)+\beta\left(P-c^{2}\right)\left[1-F\left(y^{2}-w\right)\right] .
\end{array}
$$

Note that the induction hypothesis was used to obtain the following result:

$$
\begin{aligned}
& \frac{\partial V_{n-1}\left(y^{1}-y^{2}, w+z-y^{2}\right)}{\partial y^{2}} \\
= & \lim _{\epsilon \rightarrow 0} \frac{V_{n-1}\left(y^{1}-y^{2}-\epsilon, w+z-y^{2}-\epsilon\right)-V_{n-1}\left(y^{1}-y^{2}, w+z-y^{2}\right)}{\epsilon} \\
= & \lim _{\epsilon \rightarrow 0} \frac{\left(P-c^{2}\right) \epsilon}{\epsilon}=P-c^{2} .
\end{aligned}
$$

Now, solving the first-order equation for $y^{2}$, we get

$$
F\left(y^{2}-w\right)=F\left(S_{n}^{2}\right)=\frac{\pi+P-c^{2}+h^{1}-\beta\left(P-c^{2}\right)}{\pi+P+h^{1}+h^{2}-\beta\left(P-c^{2}\right)} .
$$

Finally, we show that $v_{n}\left(x, w, y^{1}, y^{2}\right)$ is a convex function for $y^{2} \geq w$,

$$
\frac{\partial^{2} v_{n}\left(x, w, y^{1}, y^{2}\right)}{\partial\left(y^{2}\right)^{2}}=\left[\pi+P+h^{1}+h^{2}-\beta\left(P-c^{2}\right)\right] f\left(y^{2}-w\right)>0 .
$$

A similar algebra shows that $v_{n}\left(x, w, y^{1}, y^{2}\right)$ is strictly decreasing in $y^{2}$ for $y^{2} \leq w$. Combining two cases, it can be easily shown that $S_{n}^{2}$ minimizes the cost when $y^{1} \geq S_{n}^{2}+w$ while ordering the largest feasible quantity, $y^{1}$ achieves the minimum when $y^{1}<S_{n}^{2}+w$. Therefore, $y^{2}=\min \left\{y^{1}, S_{n}^{2}+w\right\}$.

(b) We follow the same procedure sketched in the proof for Period 1. First note that, if $\left(y^{1}, y^{2}\right)$ is optimal in state $(x, w)$, order-up-to levels $\left(y^{1}+\Delta, y^{2}+\Delta\right)$, must be feasible in state $(x+\Delta, w+\Delta)$. If $y^{2}>w$, the cost associated with a policy that raises inventories of both parts to $\left(y^{1}+\Delta, y^{2}+\Delta\right)$ in period $n$ and follows the optimal policy afterward is:

$$
\begin{aligned}
& v_{n}\left(x+\Delta, w+\Delta, y^{1}+\Delta, y^{2}+\Delta\right) \\
& =K \delta\left(y^{1}-x\right)^{+}+c^{1}\left(y^{1}-x\right)+c^{2}\left(y^{2}+\Delta\right) \\
& \quad+\int_{y^{2}-w}^{\infty}\left[\pi\left(z+w-y^{2}\right)+h^{1}\left(y^{1}-y^{2}\right)-P\left(y^{2}+\Delta\right)\right. \\
& \left.\quad+\beta V_{n-1}\left(y^{1}-y^{2}, w+z-y^{2}\right)\right] f(z) d z \\
& \quad+\int_{0}^{y^{2}-w}\left[h^{1}\left(y^{1}-w-z\right)+h^{2}\left(y^{2}-w-z\right)\right. \\
& \left.\quad-P(z+w+\Delta)+\beta V_{n-1}\left(y^{1}-z-w, 0\right)\right] f(z) d z \\
& =V_{n}(x, w)+\left(c^{2}-P\right) \Delta .
\end{aligned}
$$

If $y^{2} \leq w$, then the second integral disappears but the result holds. Therefore, $V_{n}(x+\Delta, w+\Delta) \leq V_{n}(x, w)+\left(c^{2}-P\right) \Delta$.

To show the inequality holds for the opposite direction, notice that if raising inventories to $\left(y^{1}, y^{2}\right)$ is optimal in state $(x+\Delta$, 
$w+\Delta)$, then $\left(y^{1}-\Delta, y^{2}-\Delta\right)$ must be feasible in state $(x, w)$. If $y^{2}-\Delta>w$, then

$$
\begin{array}{r}
v_{n}\left(x, w, y^{1}-\Delta, y^{2}-\Delta\right) \\
=K \delta\left(y^{1}-\Delta-x\right)^{+}+c^{1}\left(y^{1}-\Delta-x\right)+c^{2}\left(y^{2}-\Delta\right) \\
\quad+\int_{y^{2}-\Delta-w}^{\infty}\left[\pi\left(z+w-y^{2}+\Delta\right)+h^{1}\left(y^{1}-y^{2}\right)\right. \\
\left.-P\left(y^{2}-\Delta\right)+\beta V_{n-1}\left(y^{1}-y^{2}, w+z-y^{2}+\Delta\right)\right] f(z) d z \\
+\int_{0}^{y^{2}-\Delta-w}\left[h^{1}\left(y^{1}-\Delta-w-z\right)+h^{2}\left(y^{2}-\Delta-w-z\right)\right. \\
\left.-P(z+w)+\beta V_{n-1}\left(y^{1}-\Delta-z-w, 0\right)\right] f(z) d z \\
=V_{n}(x+\Delta, w+\Delta)-\left(c^{2}-P\right) \Delta,
\end{array}
$$

and we have $V_{n}(x, w) \leq V_{n}(x+\Delta, w+\Delta)-\left(c^{2}-P\right) \Delta$. If $y^{2}-\Delta \leq w$, then the second integral disappears but the result holds.

Since $y^{1} \geq x+\Delta$ and $y^{2}=\min \left\{y^{1}, S_{n}^{2}+w+\Delta\right\}$, if $\left(y^{1}, y^{2}\right)$ is optimal in state $(x+\Delta, w+\Delta)$, then $\left(y^{1}-\Delta, y^{2}-\Delta\right)$ must be feasible in state $(x, w)$.

Having proved both sides of the inequality, we conclude that $V_{n}(x, w)-V_{n}(x+\Delta, w+\Delta)=\left(P-c^{2}\right) \Delta$ and $\left(y^{1}+\Delta, y^{2}+\Delta\right)$ is optimal in state $(x+\Delta, w+\Delta)$.

LEMMA 1: If it is optimal to order part 1 in state $(x, w)$, then $V_{n}(x, w)-$ $V_{n}(x, w+\Delta) \geq\left(P-c^{1}-c^{2}\right) \Delta$ for $\Delta>0$.

PROOF: First note that if $\left(y^{1}, y^{2}\right)$ is optimal in state $(x, w),\left(y^{1}+\Delta, y^{2}+\right.$ $\Delta$ ) must be feasible in state $(x, w+\Delta)$. Then, we have

$$
\begin{aligned}
v_{n}(x, w+ & \left.\Delta, y^{1}+\Delta, y^{2}+\Delta\right)=K+c^{1}\left(y^{1}+\Delta-x\right) \\
& +c^{2}\left(y^{2}+\Delta\right)+\int_{y^{2}-w}^{\infty}\left[\pi\left(z+w-y^{2}\right)+h^{1}\left(y^{1}-y^{2}\right)\right. \\
& \left.\quad P\left(y^{2}+\Delta\right)+\beta V_{n-1}\left(y^{1}-y^{2}, z+w-y^{2}\right)\right] f(z) d z \\
+ & \int_{0}^{y^{2}-w}\left[h^{1}\left(y^{1}-w-z\right)+h^{2}\left(y^{2}-w-z\right)-P(z+w+\Delta)\right. \\
& \left.+\beta V_{n-1}\left(y^{1}-w-z, 0\right)\right] f(z) d z=V_{n}(x, w)+\left(c^{1}+c^{2}-P\right) \Delta .
\end{aligned}
$$

Hence, $V_{n}(x, w+\Delta) \leq V_{n}(x, w)+\left(c^{1}+c^{2}-P\right) \Delta$.

\section{LEMMA 2:}

(a) In a given period, if it is optimal to order part 1 and $x<w$, then it is optimal to order at least up to $w$. In other words, if $y^{1}>x$ and $x<w$, then $y^{1} \geq w$.

(b) $V_{n}(0, w+\Delta)-V_{n}(0, w) \geq\left(c^{1}+c^{2}-P\right) \Delta$ for $\Delta>0$ and $n \geq 1$.

PROOF: We use induction on the period $n$ to prove these two properties concurrently.

For Period 1:

(a) Suppose $y^{1}<w$, then $y^{2}=\min \left\{y^{1}, S_{1}^{2}+w\right\}=y^{1}$ in state $(x, w)$ and

$$
\begin{aligned}
V_{1}(x, w)=v_{1}\left(x, w, y^{1}, y^{1}\right) & =K+c^{1}\left(y^{1}-x\right)+c^{2} y^{1} \\
+ & \int_{0}^{\infty}\left[\pi\left(z+w-y^{1}\right)-P y^{1}\right] f(z) d z .
\end{aligned}
$$

Naval Research Logistics DOI 10.1002/nav
Comparing this to the cost when order-up-to levels, $(w, w)$, are used, we have

$$
\begin{aligned}
v_{1}(x, w, w, w) & =K+c^{1}(w-x)+c^{2} w+\int_{0}^{\infty}[\pi z-P w] f(z) d z \\
& =V_{1}(x, w)-\left(P+\pi-c^{1}-c^{2}\right)\left(w-y^{1}\right) \\
& <V_{1}(x, w) .
\end{aligned}
$$

This is a contradiction to the optimality of $\left(y^{1}, y^{1}\right)$, and hence we conclude that ordering up to $y^{1}<w$ is not optimal for period 1 .

(b) CASE 1: Not ordering is optimal in state $(0, w+\Delta)$. In this case, from Theorem 1, it is optimal to not order either part is optimal in state $(0, w+\Delta)$ (i.e., $\left.\left(y^{1}, y^{2}\right)=(0,0)\right)$. Because $(0,0)$ is also feasible in state $(0, w)$, we have

$$
\begin{aligned}
V_{1}(0, w+\Delta)- & V_{1}(0, w) \\
\geq V_{1}(0, w+\Delta)-v_{1}(0, w, 0,0) & =\pi \Delta \geq\left(c^{1}+c^{2}-P\right) \Delta .
\end{aligned}
$$

CASE 2: Suppose ordering up to $\left(y^{1}, y^{2}\right)$ is optimal in state $(0, w+\Delta)$. Since both $y^{1}$ and $y^{2}$ are greater than or equal to $\Delta$ by Theorem 1(a) and Lemma 2(a), $\left(y^{1}-\Delta, y^{2}-\Delta\right)$ must be feasible in state $(0, w)$. Hence,

$$
\begin{array}{r}
V_{1}(0, w+\Delta)-V_{1}(0, w) \\
\geq V_{1}(0, w+\Delta)-v_{1}\left(0, w, y^{1}-\Delta, y^{2}-\Delta\right) \\
=\left(c^{1}+c^{2}-P\right) \Delta .
\end{array}
$$

For Period $n-1$ : Assume:

(a) In period $n-1$, if $y^{1}>x$ and $x<w$ then $y^{1} \geq w$.

(b) $V_{n-1}(0, w+\Delta)-V_{n-1}(0, w) \geq\left(c_{n-1}^{1}+c_{n-1}^{2}-P_{n-1}\right) \Delta$.

\section{For Period $n:$}

(a) Suppose $y^{1}<w$ in state $(x, w)$. Then, $y^{2}=\min \left\{y^{1}, S_{1}^{2}+w\right\}=y^{1}$ in state $(x, w)$ and

$$
\begin{array}{r}
V_{n}(x, w)=K+c^{1}\left(y^{1}-x\right)+c^{2} y^{1}+\int_{0}^{\infty}\left[\pi\left(z+w-y^{1}\right)\right. \\
\left.-P y^{1}+\beta V_{n-1}\left(0, z+w-y^{1}\right)\right] f(z) d z .
\end{array}
$$

Since $(w, w)$ is feasible in this state, we have

$$
\begin{aligned}
& v_{n}(x, w, w, w)=K+c^{1}(w-x)+c^{2} w \\
& \quad+\int_{0}^{\infty}\left[\pi z-P w+\beta V_{n-1}(0, z)\right] f(z) d z \\
& =V_{n}(x, w)-\left(P+\pi-c^{1}-c^{2}\right)\left(w-y^{1}\right) \\
& -\beta \int_{0}^{\infty}\left[V_{n-1}\left(0, z+w-y^{1}\right)-V_{n-1}(0, z)\right] f(z) d z \\
& \leq V_{n}(x, w)-\left(P+\pi-c^{1}-c^{2}\right)\left(w-y^{1}\right)-\beta\left(c^{1}+c^{2}-P\right)\left(w-y^{1}\right) \\
& =V_{n}(x, w)-\pi\left(w-y^{1}\right)-\left[P-c^{1}-c^{2}-\beta\left(P-c^{1}-c^{2}\right)\right]\left(w-y^{1}\right) \\
& <V_{n}(x, w) .
\end{aligned}
$$

The first inequality follows by part (b) of the induction hypothesis. We conclude that ordering up to $y^{1}<w$ is not optimal. 
(b) CASE 1: Not ordering is optimal in state $(0, w+\Delta)$. In this case, from Theorem $1,\left(y^{1}, y^{2}\right)=(0,0)$ is optimal in state $(0, w+\Delta)$. Since not ordering $((0,0))$ is also feasible in state $(0, w)$, we have

$$
\begin{array}{r}
V_{n}(0, w+\Delta)-V_{n}(0, w) \geq V_{n}(0, w+\Delta)-v_{n}(0, w, 0,0) \\
=\pi \Delta+\beta \int_{0}^{\infty} \quad\left[V_{n-1}(0, z+w+\Delta)-V_{n-1}(0, z+w)\right] f(z) d z \\
\geq \pi \Delta+\beta\left(c^{1}+c^{2}-P\right) \Delta \geq\left(c^{1}+c^{2}-P\right) \Delta .
\end{array}
$$

CASE 2: Ordering is optimal in state $(0, w+\Delta)$. Suppose that $\left(y^{1}, y^{2}\right)$ are optimal order-up-to levels for parts 1 and 2, respectively, in state $(0, w+\Delta)$. Since both $y^{1}$ and $y^{2}$ are greater than or equal to $\Delta$ by Theorem 1 and Lemma $2(a),\left(y^{1}-\Delta, y^{2}-\Delta\right)$ must be feasible in state $(0, w)$. Therefore,

$$
\begin{aligned}
V_{n}(0, w+\Delta)-V_{n}(0, w) & \geq V_{n}(0, w+\Delta)-v_{n}\left(0, w, y^{1}-\Delta, y^{2}-\Delta\right) \\
& =\left(c^{1}+c^{2}-P\right) \Delta .
\end{aligned}
$$

LEMMA 3: If $y>x, x-w>0$ and $y-w>0$, then

(a) $(x-y)[F(y-w)-F(x-w)]<\int_{x-w}^{y-w}(z+w-y) f(z) d z<0$, and

(b) $(x-y)[F(y-w)-F(x-w)]<\int_{x-w}^{y-w}(x-w-z) f(z) d z<0$.

\section{PROOF:}

1. For any $z, x-w<z<y-w$, we have $x-y<z+w-y<0$. Hence,

$$
\begin{aligned}
& (x-y) \int_{x-w}^{y-w} f(z) d z<\int_{x-w}^{y-w}(z+w-y) f(z) d z<0, \\
& (x-y)[F(y-w)-F(x-w)]<\int_{x-w}^{y-w}(z+w-y) f(z) d z<0 .
\end{aligned}
$$

2. For any $z, x-w<z<y-w$, we have $x-y<x-w-z<0$. Hence,

$$
\begin{aligned}
& (x-y) \int_{x-w}^{y-w} f(z) d z<\int_{x-w}^{y-w}(x-w-z) f(z) d z<0, \\
& (x-y)[F(y-w)-F(x-w)]<\int_{x-w}^{y-w}(x-w-z) f(z) d z<0 .
\end{aligned}
$$

PROOF OF THEOREM 2: We will use induction on the period, $n$ to, prove the following properties concurrently:

(a) If it is optimal to order part 1 in state $(x, w)$, i.e. $y^{1}>x$, and the optimal order-up-to levels are $\left(y^{1}, y^{2}\right)$, then $y^{2}>x$ and $y^{2} \geq w$.

(b) If it is optimal to order part 1 in state $(x, w)$, then it is also optimal to order part 1 in state $(x, w+\Delta)$.

(c) If it is optimal to order part 1 in state $(x, w)$ and the optimal orderup-to levels are $\left(y^{1}, y^{2}\right)$ for that state is $\left(y^{1}, y^{2}\right)$, then the optimal order-up-to levels for state $(x, w+\Delta)$ are $\left(y^{1}+\Delta, y^{2}+\Delta\right)$ and $V_{n}(x, w)-V_{n}(x, w+\Delta)=\left(P-c^{1}-c^{2}\right) \Delta$.
For period 1:

(a) Suppose that it is optimal to order part 1 in state $(x, w)$. Then, by Theorem 1(a) and Lemma 2, $y^{2} \geq w$. Assume $y^{2} \leq x$. Then, we have

$$
\begin{aligned}
& v_{1}\left(x, w, x, y^{2}\right)=c^{2} y^{2}+\int_{y^{2}-w}^{\infty}\left[\pi\left(z+w-y^{2}\right)\right. \\
&\left.+h^{1}\left(x-y^{2}\right)-P y^{2}\right] f(z) d z+\int_{0}^{y^{2}-w}\left[h^{1}(x-w-z)+\right. \\
&\left.h^{2}\left(y^{2}-w-z\right)-P(z+w)\right] f(z) d z \\
&=v_{1}\left(x, w, y^{1}, y^{2}\right)-K-c^{1}\left(y^{1}-x\right)-h^{1}\left(y^{1}-x\right) \\
&<v_{1}\left(x, w, y^{1}, y^{2}\right)=V_{1}(x, w) .
\end{aligned}
$$

The fact that order-up-to levels $\left(x, y^{2}\right)$ are strictly better than $\left(y^{1}, y^{2}\right)$ contradicts the optimality of $\left(y^{1}, y^{2}\right)$, so $y^{2}>x$.

(b) In this part, we will show that if it is optimal to order part 1 in state $(x, w)$, then it is also optimal to order part 1 in state $(x, w+\Delta)$. Suppose order-up-to levels $\left(y^{1}, y^{2}\right)$ are optimal in state $(x, w)$. Since it is optimal to order in state $(x, w)$, we know that $v_{1}(x, w, x, x)>v_{1}\left(x, w, y^{1}, y^{2}\right)=V_{1}(x, w)$. Using this relation, we show that the following important result holds for $x>w$ :

$$
\begin{aligned}
& 0<v_{1}(x, w, x, x)-v_{1}\left(x, w, y^{1}, y^{2}\right) \\
& =c^{2} x-K-c^{1}\left(y^{1}-x\right)-c^{2} y^{2} \\
& +\int_{x-w}^{\infty}[\pi(z+w-x)-P x] f(z) d z+\int_{0}^{x-w}\left[\left(h^{1}+h^{2}\right)(x-w-z)\right. \\
& -P(z+w)] f(z) d z \\
& -\int_{y^{2}-w}^{\infty}\left[\pi\left(z+w-y^{2}\right)+h^{1}\left(y^{1}-y^{2}\right)-P y^{2}\right] f(z) d z \\
& -\int_{0}^{y^{2}-w}\left[h^{1}\left(y^{1}-w-z\right)+h^{2}\left(y^{2}-w-z\right)-P(z+w)\right] f(z) d z \\
& =-K-c^{1}\left(y^{1}-x\right)-c^{2}\left(y^{2}-x\right) \\
& +\int_{x-w}^{y^{2}-w}[\pi(z+w-x)-P x] f(z) d z \\
& +\int_{y^{2}-w}^{\infty}[\pi(z+w-x)-P x] f(z) d z \\
& +\int_{0}^{x-w}\left[\left(h^{1}+h^{2}\right)(x-w-z)-P(z+w)\right] f(z) d z \\
& -\int_{y^{2}-w}^{\infty}\left[\pi\left(z+w-y^{2}\right)+h^{1}\left(y^{1}-y^{2}\right)-P y^{2}\right] f(z) d z \\
& -\int_{0}^{x-w}\left[h^{1}\left(y^{1}-w-z\right)+h^{2}\left(y^{2}-w-z\right)-P(z+w)\right] f(z) d z \\
& -\int_{x-w}^{y^{2}-w}\left[h^{1}\left(y^{1}-w-z\right)+h^{2}\left(y^{2}-w-z\right)-P(z+w)\right] f(z) d z .
\end{aligned}
$$

After applying extensive algebra and Lemma 3(b), we have

$$
\begin{aligned}
0<v_{1}(x, w, x, x)-v_{1}\left(x, w, y^{1}, y^{2}\right) \\
=-K-c^{1}\left(y^{1}-x\right)-c^{2}\left(y^{2}-x\right)-h^{2}\left(y^{2}-x\right) \\
\times F(x-w)-h^{1}\left(y^{1}-y^{2}\right)-h^{1}\left(y^{2}-x\right) F(x-w) \\
+P\left(y^{2}-x\right)[1-F(x-w)]+\pi\left(y^{2}-x\right)[1-F(x-w)] \\
+\int_{x-w}^{y^{2}-w}\left[\left(\pi+P+h^{1}+h^{2}\right)\left(z+w-y^{2}\right)\right] f(z) d z \\
<-K-c^{1}\left(y^{1}-x\right)-c^{2}\left(y^{2}-x\right)+P\left(y^{2}-x\right)+\pi\left(y^{2}-x\right)
\end{aligned}
$$

Naval Research Logistics DOI 10.1002/nav 


$$
\begin{array}{r}
-\left(h^{1}+h^{2}+P+\pi\right)\left(y^{2}-x\right) F(x-w)-h^{1}\left(y^{1}-y^{2}\right) \\
=-K-\left(c^{1}+h^{1}\right)\left(y^{1}-x\right)+\left(\pi+P+h^{1}-c^{2}\right)\left(y^{2}-x\right) \\
-\left(\pi+P+h^{1}+h^{2}\right)\left(y^{2}-x\right) F(x-w) .
\end{array}
$$

From the last inequality, we obtain the following bound for $F(x-w)$ :

$$
F(x-w)<-\frac{K+\left(c^{1}+h^{1}\right)\left(y^{1}-x\right)}{\left(\pi+P+h^{1}+h^{2}\right)\left(y^{2}-x\right)}+\frac{\pi+P+h^{1}-c^{2}}{\pi+P+h^{1}+h^{2}} .
$$

Because $\left(y^{1}, y^{2}\right)$ is optimal in state $(x, w)$ then, from result (a) of this theorem $y^{2}>x$ and by Theorem 1(a), $x<y^{2} \leq S_{1}^{2}+w$. On the other hand, if not ordering part 1 were optimal in state $(x, w+\Delta)$, ordering $x$ units of part 2 only (i.e., using order-up-to levels $(x, x)$ ) must be optimal since $x<S_{1}^{2}+w<S_{1}^{2}+w+\Delta$. The cost when order-up-to levels $(x, x)$ are used in state $(x, w+\Delta)$ is:

$$
\begin{gathered}
v_{1}(x, w+\Delta, x, x)=c^{2} x+\int_{x-w-\Delta}^{\infty}[\pi(z+w+\Delta-x)-P x] f(z) d z \\
+\int_{0}^{x-w-\Delta}\left[\left(h^{1}+h^{2}\right)(x-w-\Delta-z)-P(z+w+\Delta)\right] f(z) d z \\
=v_{1}(x, w, x, x)+\left(h^{1}+h^{2}+\pi+P\right) \int_{x-w-\Delta}^{x-w}(z+w-x) f(z) d z \\
+\pi \Delta[1-F(x-w-\Delta)]-\left(h^{1}+h^{2}+P\right) \Delta F(x-w-\Delta) \\
\geq v_{1}(x, w, x, x)-\left(h^{1}+h^{2}+\pi+P\right) \Delta[F(x-w)-F(x-w-\Delta)] \\
\quad-\left(h^{1}+h^{2}+P+\pi\right) \Delta F(x-w-\Delta)+\pi \Delta \\
=v_{1}(x, w, x, x)+\pi \Delta-\left(h^{1}+h^{2}+\pi+P\right) \Delta F(x-w) \\
>v_{1}(x, w, x, x)-P \Delta-h^{1} \Delta+c^{2} \Delta+\frac{K \Delta+\left(c^{1}+h^{1}\right)\left(y^{1}-x\right) \Delta}{y^{2}-x} \\
>V_{1}(x, w)-P \Delta-h^{1} \Delta+c^{2} \Delta+\frac{K \Delta+\left(c^{1}+h^{1}\right)\left(y^{1}-x\right) \Delta}{y^{2}-x} \\
\geq V_{1}(x, w+\Delta)+\left(P-c^{1}-c^{2}\right) \Delta-P \Delta \\
-h^{1} \Delta+c^{2} \Delta+\frac{K \Delta+\left(c^{1}+h^{1}\right)\left(y^{1}-x\right) \Delta}{y^{2}-x} \\
=V_{1}(x, w+\Delta)+\frac{K \Delta+\left(c^{1}+h^{1}\right)\left(y^{1}-y^{2}\right) \Delta}{y^{2}-x} \\
>V_{1}(x, w+\Delta) .
\end{gathered}
$$

The first inequality follows from Lemma 3(a) whereas the second inequality comes directly from (T2.1). The third inequality holds due to the fact that $v_{1}(x, w, x, x)$ is not optimal and the fourth inequality comes from Lemma 1 . Therefore, not ordering part 1 cannot be optimal in state $(x, w+\Delta)$ for $x>w$.

If $x \leq w$ and not ordering part 1 were optimal in state $(x, w+\Delta)$, then $x \leq S_{1}^{2}+w<S_{1}^{2}+w+\Delta$ and the optimal order-up-to levels in state $(x, w+\Delta)$ must be $(x, x)$. Applying the fact that it is optimal to order part 1 in state $(x, w)$ is optimal and Lemma 1 , respectively, we get

$$
\begin{aligned}
v_{1}(x, w+\Delta, x, x) & =c^{2} x+\int_{0}^{\infty}[\pi(z+w+\Delta-x)-P x] f(z) d z \\
& =v_{1}(x, w, x, x)+\pi \Delta \\
& >V_{1}(x, w)+\pi \Delta \\
& \geq V_{1}(x, w+\Delta)+\left(\pi+P-c^{1}-c^{2}\right) \Delta .
\end{aligned}
$$

Because $P-c^{1}-c^{2} \geq 0, v_{1}(x, w+\Delta, x, x)>V_{1}(x, w+\Delta)$ for any $\Delta>0$, therefore it is also optimal to order part 1 in state $(x, w+\Delta)$.

(c) From Lemma 1, we have:

$$
V_{1}(x, w+\Delta) \leq V_{1}(x, w)+\left(c^{1}+c^{2}-P\right) \Delta .
$$

Therefore, it remains to show that the inequality holds for the opposite direction as well. To this end, first note that, from part (b) of this same theorem, we know it is also optimal to order part 1 in state $(x, w+\Delta)$. Suppose order-up-to levels $\left(y^{1}+\Delta, y^{2}+\Delta\right)$ are optimal in state $(x, w+\Delta)$. We now show that order-up-to levels $\left(y^{1}, y^{2}\right)$ are not only feasible, but also optimal in state $(x, w)$. To show that $\left(y^{1}, y^{2}\right)$ is feasible, it suffices to show that $y^{1}>x$ (later we will show that $y^{1}>x$ is always true) as $y^{2} \geq w$ from part (a) of this theorem. Provided that $\left(y^{1}, y^{2}\right)$ is feasible in state $(x, w)$, the result is immediate since

$$
\begin{aligned}
& v_{1}\left(x, w, y^{1}, y^{2}\right)=K+c^{1}\left(y^{1}-x\right)+c^{2} y^{2} \\
& +\int_{y^{2}-w}^{\infty}\left[\pi\left(z+w-y^{2}\right)+h^{1}\left(y^{1}-y^{2}\right)-P y^{2}\right] f(z) d z \\
& +\int_{0}^{y^{2}-w}\left[h^{1}\left(y^{1}-w-z\right)+h^{2}\left(y^{2}-w-z\right)-P(z+w)\right] f(z) d z \\
& =V_{1}(x, w+\Delta)-\left(c^{1}+c^{2}-P\right) \Delta,
\end{aligned}
$$

and we have

$$
V_{1}(x, w) \leq V_{1}(x, w+\Delta)+\left(P-c^{1}-c^{2}\right) \Delta .
$$

We now show that $y^{1}>x$. To do so, we need to prove that if it is optimal to order part 1 in state $(x, w+\Delta)$, then ordering part 1 up to any quantity less than $x+\Delta$ cannot be optimal. We will complete this proof by contradiction. Assume $V_{1}(x, w+\Delta)=$ $v_{1}\left(x, w+\Delta, x+\delta, y^{2^{\prime}}\right)$ for some $\delta$ such that $0 \leq \delta \leq \Delta$. By part (a) of this theorem and Theorem 1(a), $x+\delta \geq y^{2^{\prime}} \geq w+\Delta$, so $x \geq \Delta-\delta+w \geq \Delta-\delta$. Hence, $(x, w+\Delta-\delta)$ and $(x+\delta-\Delta, w)$ are valid states, and ordering up to $\left(x+\delta-\Delta, y^{2^{\prime}}-\Delta\right)$ is feasible in state $(x+\delta-\Delta, w)$. From Lemma 1 ,

$$
\begin{aligned}
& V_{1}(x, w+\Delta) \leq V_{1}(x, w+\Delta-\delta)+\left(c^{1}+c^{2}-P\right) \delta \\
& \quad=V_{1}(x+\delta-\Delta, w)-\left(P-c^{2}\right)(\Delta-\delta)+\left(c^{1}+c^{2}-P\right) \delta \\
& \quad \leq v_{1}\left(x+\delta-\Delta, w, x+\delta-\Delta, y^{2^{\prime}}-\Delta\right)+c^{1} \delta+c^{2} \Delta-P \Delta .
\end{aligned}
$$

We note that the first equality is obtained from Theorem 1(b) whereas the second inequality comes from the fact that the optimal cost $V_{1}(x+\delta-\Delta, w)$ is less than or equal to the cost when order-up-to levels $\left(x+\delta-\Delta, y^{2^{\prime}}-\Delta\right)$ are used. However,

$$
\begin{aligned}
& V_{1}(x, w+\Delta)=v_{1}\left(x, w+\Delta, x+\delta, y^{2^{\prime}}\right) \\
& =K+c^{1} \delta+c^{2} y^{2^{\prime}}+\int_{y^{2^{\prime}}-w-\Delta}^{\infty}\left[\pi\left(z+w+\Delta-y^{2^{\prime}}\right)\right. \\
& \left.+h^{1}\left(x+\delta-y^{2^{\prime}}\right)-P y^{2^{\prime}}\right] f(z) d z \\
& +\int_{0}^{y^{2^{\prime}}-w-\Delta}\left[h^{1}(x+\delta-w-\Delta-z)+h^{2}\left(y^{2^{\prime}}-w-\Delta-z\right)\right. \\
& \quad P(z+w+\Delta)] f(z) d z \\
& =v_{1}\left(x+\delta-\Delta, w, x+\delta-\Delta, y^{2^{\prime}}-\Delta\right)+K+c^{1} \delta+c^{2} \Delta-P \Delta .
\end{aligned}
$$


This identity contradicts the inequality (T2.4) as $V_{1}(x, w+\Delta)>$ $v_{1}\left(x+\delta-\Delta, w, x+\delta-\Delta, y^{2^{\prime}}-\Delta\right)+c^{1} \delta+c^{2} \Delta-P \Delta$. Hence, it cannot be optimal to order less than $x+\Delta$ in state $(x, w+\Delta)$ and (T2.3) is always true.

From (T2.2) and (T2.3), $V_{1}(x, w)=V_{1}(x, w+\Delta)+\left(P-c^{1}-\right.$ $\left.c^{2}\right) \Delta$, and $\left(y^{1}+\Delta, y^{2}+\Delta\right)$ is optimal in state $(x, w+\Delta)$.

\section{For period $n-1$ : Assume}

(a) If it is optimal to order part 1 in state $(x, w)$ and the optimal order-up-to levels are $\left(y^{1}, y^{2}\right)$, then $y^{2}>x$ and $y^{2} \geq w$.

(b) If it is optimal to order part 1 in state $(x, w)$, then it is also optimal to order part 1 in state $(x, w+\Delta)$.

(c) If it is optimal to order part 1 in state $(x, w)$ and the optimal order-up-to levels for that state is $\left(y^{1}, y^{2}\right)$, then the optimal order-up-to levels for state $(x, w+\Delta)$ is $\left(y^{1}+\Delta, y^{2}+\Delta\right)$ and $V_{n-1}(x, w)-V_{n-1}(x, w+\Delta)=\left(P-c^{1}-c^{2}\right) \Delta$.

For period $n$ :

(a) Under the assumption that it is optimal to order part 1 in state $(x, w)$, by Theorem 1(a) and Lemma 2, $y^{2} \geq w$. Assume $y^{2} \leq x$. On the other hand, if we do not order part 1 , but order $y^{2}$ units of part 2, we have

$$
\begin{gathered}
v_{n}\left(x, w, x, y^{2}\right) \\
=c^{2} y^{2}+\int_{y^{2}-w}^{\infty}\left[\pi\left(z+w-y^{2}\right)+h^{1}\left(x-y^{2}\right)-P y^{2}\right. \\
\left.+\beta V_{n-1}\left(x-y^{2}, w+z-y^{2}\right)\right] f(z) d z \\
+\int_{0}^{y^{2}-w}\left[h^{1}(x-w-z)+h^{2}\left(y^{2}-w-z\right)-P(z+w)\right. \\
\left.\quad+\beta V_{n-1}(x-w-z, 0)\right] f(z) d z \\
=v_{n}\left(x, w, y^{1}, y^{2}\right)-K-c^{1}\left(y^{1}-x\right)-h^{1}\left(y^{1}-x\right) \\
\beta\left[V_{n-1}\left(y^{1}-y^{2}, w+z-y^{2}\right)-V_{n-1}\left(x-y^{2}, w+z-y^{2}\right)\right] f(z) d z \\
-\int_{0}^{y^{2}-w} \beta\left[V_{n-1}\left(y^{1}-w-z, 0\right)-V_{n-1}(x-w-z, 0)\right] f(z) d z .
\end{gathered}
$$

Applying Theorem 1(b), we rewrite $V_{n-1}(x-w-z, 0)$ and $V_{n-1}\left(x-y^{2}, w+z-y^{2}\right)$ as follows: $V_{n-1}(x-w-z, 0)=$ $V_{n-1}\left(y^{1}-w-z, y^{1}-x\right)+\left(P-c^{2}\right)\left(y^{1}-x\right)$, and $V_{n-1}\left(x-y^{2}, w+\right.$ $\left.z-y^{2}\right)=V_{n-1}\left(y^{1}-y^{2}, w+z-y^{2}+y^{1}-x\right)+\left(P-c^{2}\right)\left(y^{1}-x\right)$.

Then,

$$
\begin{gathered}
v_{n}\left(x, w, x, y^{2}\right)=v_{n}\left(x, w, y^{1}, y^{2}\right)-K-c^{1}\left(y^{1}-x\right)-h^{1}\left(y^{1}-x\right) \\
-\int_{y^{2}-w}^{\infty} \beta\left[V_{n-1}\left(y^{1}-y^{2}, w+z-y^{2}\right)-V_{n-1}\left(y^{1}-y^{2}, w+z-y^{2}\right.\right. \\
\left.\left.+y^{1}-x\right)-\left(P-c^{2}\right)\left(y^{1}-x\right)\right] f(z) d z \\
-\int_{0}^{y^{2}-w} \beta\left[V_{n-1}\left(y^{1}-w-z, 0\right)-V_{n-1}\left(y^{1}-w-z, y^{1}-x\right)\right. \\
\left.-\left(P-c^{2}\right)\left(y^{1}-x\right)\right] f(z) d z \\
=v_{n}\left(x, w, y^{1}, y^{2}\right)-K-c^{1}\left(y^{1}-x\right)-h^{1}\left(y^{1}-x\right)
\end{gathered}
$$

$$
\begin{array}{r}
-\int_{y^{2}-w}^{\infty} \beta\left[\left(P-c^{1}-c^{2}\right)\left(y^{1}-x\right)-\left(P-c^{2}\right)\left(y^{1}-x\right)\right] f(z) d z \\
-\int_{0}^{y^{2}-w} \beta\left[\left(P-c^{1}-c^{2}\right)\left(y^{1}-x\right)-\left(P-c^{2}\right)\left(y^{1}-x\right)\right] f(z) d z \\
=v_{n}\left(x, w, y^{1}, y^{2}\right)-K-c^{1}\left(y^{1}-x\right)-h^{1}\left(y^{1}-x\right)+\beta c^{1}\left(y^{1}-x\right) \\
=v_{n}\left(x, w, y^{1}, y^{2}\right)-K-\left(c^{1}-\beta c^{1}\right)\left(y^{1}-x\right)-h^{1}\left(y^{1}-x\right) \\
<v_{n}\left(x, w, y^{1}, y^{2}\right)=V_{n}(x, w) .
\end{array}
$$

This contradicts the fact that $\left(y^{1}, y^{2}\right)$ is optimal, so $y^{2}>x$.

(b) In this part, we will show that, if it is optimal to order part 1 in state $(x, w)$, then it is also optimal to order part 1 in state $(x, w+\Delta)$. Suppose $\left(y^{1}, y^{2}\right)$ are the optimal order-up-to levels for parts 1 and 2 in state $(x, w)$. Since it is optimal to order part 1 in state $(x, w)$, we know that $v_{n}(x, w, x, x)>v_{n}\left(x, w, y^{1}, y^{2}\right)=V_{n}(x, w)$. Using this relation and a similar algebra used when $n=1$, we develop the following important result for $x>w$ :

$$
\begin{aligned}
& 0<v_{n}(x, w, x, x)-v_{n}\left(x, w, y^{1}, y^{2}\right) \\
& =c^{2} x+\int_{x-w}^{\infty}\left[\pi(z+w-x)-P x+\beta V_{n-1}(0, z+w-x)\right] f(z) d z \\
& +\int_{0}^{x-w}\left[\left(h^{1}+h^{2}\right)(x-w-z)-P(z+w)\right. \\
& \left.+\beta V_{n-1}(x-w-z, 0)\right] f(z) d z-K-c^{1}\left(y^{1}-x\right)-c^{2} y^{2} \\
& -\int_{y^{2}-w}^{\infty}\left[\pi\left(z+w-y^{2}\right)+h^{1}\left(y^{1}-y^{2}\right)-P y^{2}\right. \\
& \left.\left.+\beta V_{n-1}\left(y^{1}-y^{2}, z+w-y^{2}\right)\right)\right] f(z) d z \\
& -\int_{0}^{y^{2}-w}\left[h^{1}\left(y^{1}-w-z\right)+h^{2}\left(y^{2}-w-z\right)-P(z+w)\right. \\
& \left.+\beta V_{n-1}\left(y^{1}-w-z, 0\right)\right] f(z) d z \\
& =-K-c^{1}\left(y^{1}-x\right)-c^{2}\left(y^{2}-x\right)-h^{2}\left(y^{2}-x\right) F(x-w)-h^{1}\left(y^{1}-y^{2}\right) \\
& -h^{1}\left(y^{2}-x\right) F(x-w)+P\left(y^{2}-x\right)[1-F(x-w)] \\
& +\pi\left(y^{2}-x\right)[1-F(x-w)] \\
& +\int_{x-w}^{y^{2}-w}\left[\left(\pi+P+h^{1}+h^{2}\right)\left(z+w-y^{2}\right)\right] f(z) d z \\
& -\int_{x-w}^{y^{2}-w} \beta\left[V_{n-1}\left(y^{1}-w-z, 0\right)-V_{n-1}(0, z+w-x)\right] f(z) d z \\
& -\int_{y^{2}-w}^{\infty} \beta\left[V_{n-1}\left(y^{1}-y^{2}, z+w-y^{2}\right)-V_{n-1}(0, z+w-x)\right] f(z) d z \\
& -\int_{0}^{x-w} \beta\left[V_{n-1}\left(y^{1}-w-z, 0\right)-V_{n-1}(x-w-z, 0)\right] f(z) d z \\
& <-K-c^{1}\left(y^{1}-x\right)-c^{2}\left(y^{2}-x\right)+P\left(y^{2}-x\right)+\pi\left(y^{2}-x\right) \\
& -\left(h^{1}+h^{2}+P+\pi\right)\left(y^{2}-x\right) F(x-w)-h^{1}\left(y^{1}-y^{2}\right) \\
& -\int_{x-w}^{y^{2}-w} \beta\left[V_{n-1}\left(y^{1}-w-z, 0\right)-V_{n-1}\left(y^{1}-w-z, y^{1}-x\right)\right. \\
& \left.+\left(c^{2}-P\right)\left(y^{1}-w-z\right)\right] f(z) d z \\
& -\int_{y^{2}-w}^{\infty} \beta\left[V_{n-1}\left(y^{1}-y^{2}, z+w-y^{2}\right)-V_{n-1}\left(y^{1}-y^{2},\right.\right. \\
& \left.\left.z+w-y^{2}+y^{1}-x\right)\right] f(z) d z-\int_{y^{2}-w}^{\infty}\left[\left(c^{2}-P\right)\left(y^{1}-y^{2}\right)\right] f(z) d z
\end{aligned}
$$

Naval Research Logistics DOI 10.1002/nav 


$$
\begin{gathered}
-\int_{0}^{x-w} \beta\left[V_{n-1}\left(y^{1}-w-z, 0\right)-V_{n-1}\left(y^{1}-w-z, y^{1}-x\right)\right. \\
\left.+\left(c^{2}-P\right)\left(y^{1}-x\right)\right] f(z) d z \\
=-K-\left(c^{1}+h^{1}\right)\left(y^{1}-x\right)+\left(\pi+P-c^{2}+h^{1}\right)\left(y^{2}-x\right) \\
-\left(h^{1}+h^{2}+P+\pi\right)\left(y^{2}-x\right) F(x-w) \\
+\int_{x-w}^{y^{2}-w} \beta\left[c^{1}\left(y^{1}-x\right)+\left(P-c^{2}\right)(x-w-z)\right] f(z) d z \\
+\int_{y^{2}-w}^{\infty} \beta\left[c^{1}\left(y^{1}-x\right)-\left(P-c^{2}\right)\left(y^{2}-x\right)\right] f(z) d z+ \\
\quad \int_{0}^{x-w} \beta c^{1}\left(y^{1}-x\right) f(z) d z \\
<-K-\left(c^{1}-\beta c^{1}+h^{1}\right)\left(y^{1}-x\right)+\left[\pi+P+h^{1}-c^{2}\right. \\
\left.-\beta\left(P-c^{2}\right)\right]\left(y^{2}-x\right) \\
-\left[\pi+P+h^{1}+h^{2}-\beta\left(P-c^{2}\right)\right]\left(y^{2}-x\right) F(x-w) .
\end{gathered}
$$

Therefore,

$$
\begin{aligned}
F(x-w)<- & -\frac{K+\left(c^{1}-\beta c^{1}+h^{1}\right)\left(y^{1}-x\right)}{\left[\pi+P+h^{1}+h^{2}-\beta\left(P-c^{2}\right)\right]\left(y^{2}-x\right)} \\
& +\frac{\pi+P+h^{1}-c^{2}-\beta\left(P-c^{2}\right)}{\pi+P+h^{1}+h^{2}-\beta\left(P-c^{2}\right)} .
\end{aligned}
$$

In the proof above mentioned, most equalities and inequalities come directly from algebra or calculus with exceptions of a few. The second inequality is the result of Lemma 3(a) and Theorem 1, part 2. Then the induction hypothesis on claim (c) for period $n-1$ is used to get the closed form expressions for the differences between the cost-to-go functions and to establish the equality. Finally, in the last inequality, Lemma 3(b) is applied.

Because $\left(y^{1}, y^{2}\right)$ is optimal in state $(x, w)$ then, from part (a) of this theorem, $y^{2}>x$ and by Theorem 1(a) $x<y^{2} \leq S_{n}^{2}+w$. If not ordering part 1 were optimal in state $(x, w+\Delta)$, ordering $x$ units of part 2 only (i.e., order-up-to levels $(x, x)$ are used) must be optimal since $x<S_{n}^{2}+w<S_{n}^{2}+w+\Delta$. The cost from state $(x, w)$ with $n$ periods-to-go when order-up-to levels are $(x, x)$ in period $n$ and determined optimally afterward, $v_{n}(x, w+\Delta, x, x)$, is

$$
\begin{aligned}
& v_{n}(x, w+\Delta, x, x) \\
& =c^{2} x+\int_{x-w-\Delta}^{\infty}[\pi(z+w+\Delta-x)-P x \\
& \left.+\beta V_{n-1}(0, z+w+\Delta-x)\right] f(z) d z \\
& +\int_{0}^{x-w-\Delta}\left[\left(h^{1}+h^{2}\right)(x-w-\Delta-z)-P(z+w+\Delta)\right. \\
& \left.+\beta V_{n-1}(x-w-\Delta-z, 0)\right] f(z) d z \\
& =v_{n}(x, w, x, x)+\left(h^{1}+h^{2}+\pi+P\right) \int_{x-w-\Delta}^{x-w}(z+w-x) f(z) d z \\
& +\pi \Delta[1-F(x-w-\Delta)]-\left(h^{1}+h^{2}+P\right) \Delta F(x-w-\Delta) \\
& +\beta \int_{x-w-\Delta}^{x-w}\left[V_{n-1}(0, z+w-x+\Delta)-V_{n-1}(x-z-w, 0)\right] f(z) d z \\
& +\beta \int_{x-w}^{\infty}\left[V_{n-1}(0, z+w-x+\Delta)-V_{n-1}(0, z+w-x)\right] f(z) d z \\
& +\beta \int_{0}^{x-w-\Delta}\left[V_{n-1}(x-z-w-\Delta)-V_{n-1}(x-z-w, 0)\right] f(z) d z .
\end{aligned}
$$

Again, we make use of Theorem 1(b) and part (c) for period $n-1$ of this theorem (provided by induction hypothesis) to establish the following identities:

$$
\begin{aligned}
& V_{n-1}(0, z+w-x+\Delta)-V_{n-1}(x-w-z, 0) \\
& =V_{n-1}(x-w-z, \Delta)+\left(P-c^{2}\right)(x-w-z)-V_{n-1}(x-w-z, 0) \\
& =-\left(P-c^{2}\right)(w+z-x)+\left(c^{1}+c^{2}-P\right) \Delta, \\
& =V_{n-1}(0, z+w-x+\Delta)-V_{n-1}(0, z+w-x)=\left(c^{1}+c^{2}-P\right) \Delta, \text { and } \\
& V_{n-1}(x-w-z-\Delta, 0)-V_{n-1}(x-w-z, 0) \\
& =V_{n-1}(x-w-z, \Delta)+\left(P-c^{2}\right) \Delta-V_{n-1}(x-w-z, 0) \\
& =\left(P-c^{2}\right) \Delta+\left(c^{1}+c^{2}-P\right) \Delta=c^{1} \Delta .
\end{aligned}
$$

Substituting the corresponding expressions in equation (T2.6), we have:

$$
\begin{gathered}
v_{n}(x, w+\Delta, x, x) \\
\geq v_{n}(x, w, x, x)-\left(h^{1}+h^{2}+\pi+P\right) \Delta[F(x-w)-F(x-w-\Delta)] \\
-\left(h^{1}+h^{2}+\pi+P\right) \Delta F(x-w-\Delta)+\pi \Delta \\
+\beta\left[\left(P-c^{2}\right) \Delta+\left(c^{1}+c^{2}-P\right) \Delta\right][F(x-w)-F(x-w-\Delta)] \\
+\beta\left(c^{1}+c^{2}-P\right) \Delta[1-F(x-w)]+\beta c^{1} \Delta F(x-w-\Delta) \\
=v_{n}(x, w, x, x)+\pi \Delta-\left[h^{1}+h^{2}+\pi+P-\beta\left(P-c^{2}\right)\right] \Delta F(x-w) \\
\geq v_{n}(x, w, x, x)-P \Delta-h^{1} \Delta+c^{2} \Delta+\beta\left(P-c^{2}\right) \\
+\frac{K \Delta+\left(c^{1}-\beta c^{1}+h^{1}\right)\left(y^{1}-x\right) \Delta}{y^{2}-x} \\
>V_{n}(x, w)-P \Delta-h^{1} \Delta+c^{2} \Delta+\beta\left(P-c^{2}\right) \\
+\frac{K \Delta+\left(c^{1}-\beta c^{1}+h^{1}\right)\left(y^{1}-x\right) \Delta}{y^{2}-x} \\
\geq V_{n}(x, w+\Delta)+\left(P-c^{1}-c^{2}\right) \Delta-P \Delta-h^{1} \Delta+c^{2} \Delta+\beta\left(P-c^{2}\right) \\
+\frac{K \Delta+\left((1-\beta) c^{1}+h^{1}\right)\left(y^{1}-x\right) \Delta}{y^{2}-x} \\
+\beta\left(P-c^{1}-c^{2}\right)\left(y^{2}-x\right) \Delta>V_{n}(x, w+\Delta) .
\end{gathered}
$$

The second inequality comes from (T2.5), the third one from the fact that $v_{n}(x, w+\Delta, x, x)$ is not optimal, and the fourth one from Lemma 1 . Therefore, not ordering part 1 cannot be optimal in state $(x, w+\Delta)$ for $x>w$.

If $x \leq w$ and not ordering part 1 were optimal in state $(x, w+\Delta)$, then $x \leq S_{n}^{2}+w<S_{n}^{2}+w+\Delta$ and order-up-to levels $(x, x)$ must be optimal in $(x, x)$.

$$
\begin{aligned}
v_{n}(x, w+\Delta, x, x)= & c^{2} x+\int_{0}^{\infty}[\pi(z+w+\Delta-x)-P x \\
& \left.+\beta V_{n-1}(0, w+z-x)\right] f(z) d z \\
= & v_{n}(x, w, x, x)+\pi \Delta>V_{n}(x, w)+\pi \Delta \\
\geq & V_{n}(x, w+\Delta)+\left(\pi+P-c^{1}-c^{2}\right) \Delta,
\end{aligned}
$$

and we have $v_{n}(x, w+\Delta, x, x)>V_{n}(x, w+\Delta)$. Therefore, it is optimal to order part 1 in state $(x, w+\Delta)$. 
(c) From Lemma 1, we have:

$$
V_{n}(x, w+\Delta) \leq V_{n}(x, w)+\left(c^{1}+c^{2}-P\right) \Delta .
$$

From part (b) of this same theorem, we know that it is also optimal to order part 1 in state $(x, w+\Delta)$. Let $\left(y^{1}+\Delta, y^{2}+\Delta\right)$ be the optimal order-up-to levels in state $(x, w+\Delta)$. Using an argument similar to the one used in the case $n=1$, we now show that ordering up to $\left(y^{1}, y^{2}\right)$ is not only feasible, but also optimal in state $(x, w)$. Assuming $\left(y^{1}, y^{2}\right)$ is feasible, then,

$$
\begin{aligned}
& v_{n}\left(x, w, y^{1}, y^{2}\right)=K+c^{1}\left(y^{1}-x\right)+c^{2} y^{2} \\
& +\int_{y^{2}-w}^{\infty}\left[\pi\left(z+w-y^{2}\right)+h^{1}\left(y^{1}-y^{2}\right)-P y^{2}\right. \\
& \left.+\beta V_{n-1}\left(y^{1}-y^{2}, w+z-y^{2}\right)\right] f(z) d z \\
& +\int_{0}^{y^{2}-w}\left[h^{1}\left(y^{1}-w-z\right)+h^{2}\left(y^{2}-w-z\right)-P(z+w)\right. \\
& \left.+\beta V_{n-1}\left(y^{1}-z-w, 0\right)\right] f(z) d z \\
& =V_{n}(x, w+\Delta)-\left(c^{1}+c^{2}-P\right) \Delta,
\end{aligned}
$$

and we have

$$
V_{n}(x, w) \leq V_{n}(x, w+\Delta)+\left(P-c^{1}-c^{2}\right) \Delta .
$$

To show that $\left(y^{1}, y^{2}\right)$ is feasible, we first note that $y^{2} \geq w$ is a trivial implication of part (a) of this theorem. We now show that $y^{1}>x$. To do so, we need to prove that if it is optimal to order part 1 in state $(x, w+\Delta)$ then ordering part 1 up to any quantity less than $x+\Delta$ cannot be optimal. We will complete this proof by contradiction. Assume $V_{n}(x, w+\Delta)=v_{n}\left(x, w+\Delta, x+\delta, y^{2^{\prime}}\right)$ for a $\delta$ such that $0 \leq \delta \leq \Delta$. By part (a) of this theorem and Theorem 1 (a), $x+\delta \geq \bar{y}^{2^{\prime}} \geq w+\Delta$, so $x \geq \Delta-\delta+w \geq \Delta-\delta$. Hence, $(x, w+\Delta-\delta)$ and $(x+\delta-\Delta, w)$ are valid states, and order-up-to levels $\left(x+\delta-\Delta, y^{2^{\prime}}-\Delta\right)$ are feasible in state $(x+\delta-\Delta, w)$. From Lemma 1,

$$
\begin{aligned}
& V_{n}(x, w+\Delta) \leq V_{n}(x, w+\Delta-\delta)+\left(c^{1}+c^{2}-P\right) \delta \\
& \quad=V_{n}(x+\delta-\Delta, w)-\left(P-c^{2}\right)(\Delta-\delta)+\left(c^{1}+c^{2}-P\right) \delta \\
& \quad \leq v_{n}\left(x+\delta-\Delta, w, x+\delta-\Delta, y^{2^{\prime}}-\Delta\right)+c^{1} \delta+c^{2} \Delta-P \Delta
\end{aligned}
$$

The equality is obtained from Theorem 1(b). The second inequality comes from the fact that the optimal cost $V_{n}(x+\delta-\Delta, w)$ is less than or equal to $v_{n}\left(x+\delta-\Delta, w, x+\delta-\Delta, y^{2^{\prime}}-\Delta\right)$. However,

$$
\begin{aligned}
& V_{n}(x, w+\Delta)=v_{n}\left(x, w+\Delta, x+\delta, y^{2^{\prime}}\right)=K+c^{1} \delta+c^{2} y^{2^{\prime}} \\
& +\int_{y^{2^{\prime}}-w-\Delta}^{\infty}\left[\pi\left(z+w+\Delta-y^{2^{\prime}}\right)+h^{1}\left(x+\delta-y^{2^{\prime}}\right)-P y^{2^{\prime}}\right. \\
& \left.+\beta V_{n-1}\left(x+\delta-y^{2^{\prime}}, w+z+\Delta-y^{2^{\prime}}\right)\right] f(z) d z \\
& +\int_{0}^{y^{2^{\prime}}-w-\Delta}\left[h^{1}(x+\delta-w-\Delta-z)+h^{2}\left(y^{2^{\prime}}-w-\Delta-z\right)\right. \\
& \left.-P(z+w+\Delta)+\beta V_{n-1}(x+\delta-w-\Delta-z, 0)\right] f(z) d z \\
& =v_{n}\left(x+\delta-\Delta, w, x+\delta-\Delta, y^{2^{\prime}}-\Delta\right)+K+c^{1} \delta+c^{2} \Delta-P \Delta \\
& V_{n}(x, w+\Delta)>v_{n}\left(x+\delta-\Delta, w, x+\delta-\Delta, y^{2^{\prime}}-\Delta\right)+c^{1} \delta+ \\
& c^{2} \Delta-P \Delta \text { is a contradiction to (T2.9). Hence, it cannot be optimal } \\
& \text { to order less than } x+\Delta \text { in state }(x, w+\Delta) \text { and (T2.8) is always } \\
& \text { true. }
\end{aligned}
$$

\section{ACKNOWLEDGMENTS}

This work was supported by the National Science Foundation under grants DMI-0200439, DMI-9501740, and DMI0100511 .

\section{REFERENCES}

[1] A. Federgruen and P. Zipkin, An inventory model with limited production capacity and uncertain demands. I. The discountedcost criterion, Math Oper Res 11 (1986), 208-215.

[2] S. Nahmias, Perishable inventory theory: A review, Oper Res 30 (1982), 680-708.

[3] S. Nahmias and W.P. Pierskalla, A two-product perishable/nonperishable inventory problem, SIAM J Appl Math 30 (1976), 483-500.

[4] S. Nahmias, D. Perry, and W. Stadje, Perishable inventory theory: A revisitation after 20 years, Inter Conf Global Supply Chain Management, Beijing, China, August 2002.

[5] K. Rosling, Optimal inventory policies for assembly systems under random demands, Oper Res 37 (1989), 565579.

[6] C.P. Schmidt and S. Nahmias, Optimal policy for a two-stage assembly system under random demand, Oper Res 33 (1985), $1130-1145$ 